\title{
Immobilization after injury alters extracellular matrix and stem cell fate
}

\author{
Amanda K. Huber, ${ }^{1}$ Nicole Patel, ${ }^{1}$ Chase A. Pagani, ${ }^{1}$ Simone Marini, ${ }^{1}$ Karthik R. Padmanabhan, ${ }^{2}$ Daniel L. Matera, ${ }^{3}$ \\ Mohamed Said, ${ }^{3}$ Charles Hwang, ${ }^{1}$ Ginny Ching-Yun Hsu, ${ }^{4}$ Andrea A. Poli, ${ }^{5}$ Amy L. Strong, ${ }^{1}$ Noelle D. Visser, ${ }^{1}$ \\ Joseph A. Greenstein, ${ }^{1}$ Reagan Nelson, ${ }^{1}$ Shuli Li, ${ }^{1}$ Michael T. Longaker, ${ }^{6}$ Yi Tang, ${ }^{7}$ Stephen J. Weiss, ${ }^{7}$ \\ Brendon M. Baker, ${ }^{3}$ Aaron W. James, ${ }^{4}$ and Benjamin Levi ${ }^{1}$ \\ 'Section of Plastic Surgery, Department of Surgery, 'Epigenomics Core, and ${ }^{3}$ Biomedical Engineering, University of Michigan, Ann Arbor, Michigan, USA. ${ }^{4}$ Department of Pathology, Johns Hopkins University, \\ Baltimore, Maryland, USA. ${ }^{5}$ Department of Mechanical Engineering, University of Michigan, Ann Arbor, Michigan, USA. ${ }^{6}$ nstitute for Stem Cell Biology and Regenerative Medicine, Division of Plastic and \\ Reconstructive Surgery, Department of Surgery, Stanford University, Stanford, California, USA. ㄴife Sciences Institute, University of Michigan, Ann Arbor, Michigan, USA.
}

\begin{abstract}
Cells sense the extracellular environment and mechanical stimuli and translate these signals into intracellular responses through mechanotransduction, which alters cell maintenance, proliferation, and differentiation. Here we use a mouse model of trauma-induced heterotopic ossification $(\mathrm{HO})$ to examine how cell-extrinsic forces impact mesenchymal progenitor cell (MPC) fate. After injury, single-cell (sc) RNA sequencing of the injury site reveals an early increase in MPC genes associated with pathways of cell adhesion and ECM-receptor interactions, and MPC trajectories to cartilage and bone. Immunostaining uncovers active mechanotransduction after injury with increased focal adhesion kinase signaling and nuclear translocation of transcriptional coactivator TAZ, inhibition of which mitigates HO. Similarly, joint immobilization decreases mechanotransductive signaling, and completely inhibits HO. Joint immobilization decreases collagen alignment and increases adipogenesis. Further, scRNA sequencing of the $\mathrm{HO}$ site after injury with or without immobilization identifies gene signatures in mobile MPCs correlating with osteogenesis, and signatures from immobile MPCs with adipogenesis. scATAC-seq in these same MPCs confirm that in mobile MPCs, chromatin regions around osteogenic genes are open, whereas in immobile MPCs, regions around adipogenic genes are open. Together these data suggest that joint immobilization after injury results in decreased ECM alignment, altered MPC mechanotransduction, and changes in genomic architecture favoring adipogenesis over osteogenesis, resulting in decreased formation of $\mathrm{HO}$.
\end{abstract}

\section{Introduction}

By far the most common injuries seen in emergency rooms in the United States are those of the extremities $(1,2)$, accounting for $30 \%-40 \%$ of visits (1-3). The most commonly recommended intervention for extremity trauma is immobilization (4). However, while immobilization has been described to protect the injury site, reduce pain (5), and improve healing (6-8), the mechanism by which immobilization imparts these effects remains unknown.

Interactions of the extracellular matrix (ECM), a complex dynamic network, with tissue-resident multipotent mesenchymal progenitor cells (MPCs), play important roles in MPC maintenance, proliferation, and differentiation (9). The ability of the MPC to sense an extracellular mechanical stimulus as well as to translate this into an intracellular response through downstream signaling events is known as mechanotransduction (10). One such mechanotransduction pathway is mediated by specific components of the focal adhesion complex (11). Focal adhesions (FAs)

Authorship note: NP and CAP contributed equally.

Conflict of interest: The authors have declared that no conflict of interest exists. Copyright: () 2020, American Society for Clinical Investigation.

Submitted: January 2, 2020; Accepted: July 9, 2020; Published: September 21, 2020

Reference information: J Clin Invest. 2020;130(10):5444-5460.

https://doi.org/10.1172/JCl136142. link the ECM to the internal actin cytoskeleton via receptor-integrin interactions with the ECM outside and integrin-adaptor protein interactions inside, recruiting focal adhesion kinase (FAK) and orchestrating downstream signaling events. In addition to FAK signaling, other downstream pathways of mechanotransduction are important. For example, signaling through the transcriptional coactivators Yes-associated protein (YAP1) and transcriptional coactivator with PDZ-binding motif (TAZ; YAP/TAZ) have been shown as important mediators of cell differentiation in response to mechanical properties of the ECM.

Because of the difficulties of manipulating the ECM properties in vivo, including composition and alignment, many studies have relied on in vitro techniques, including 3D hydrogels, that mimic tissue elasticity. However, these systems are artificial and do not properly recapitulate the complex in vivo microenvironment present after tissue injury. A clear understanding of how the ECM exerts biomechanical cues in vivo on MPC cell fate during wound healing is needed.

In this study, we use a mouse model of trauma-induced aberrant ectopic bone formation (12) to examine the role of mechanotransduction on MPC fate in adult mice. Our group and others have demonstrated that cells of the mesenchymal lineage are responsible for heterotopic ossification (HO) (12-16). HO most frequently forms within tendons and ligaments - sites of move- 
ment and mechanical stress (17-19). In this study, we describe how cell-intrinsic forces generated as a result of altered ECM and mechanotransductive signaling after joint immobilization impact MPC fate. Single-cell sequencing (scRNA) of the MPCs from the injury site revealed an early increase in genes in pathways associated with cell adhesion and ECM-receptor interactions. Genetic deletion of the gene that encodes FAK (Ptk2) and YAP/TAZ (Yap1 and Wwtr1) in MPC lineage cells or pharmacologic FAK inhibition mitigated aberrant cell fate or HO. Joint immobilization decreased mechanotransduction signaling and resulted in a near complete inhibition of $\mathrm{HO}$ independent of tissue stiffness, accompanied by an increase in adipogenesis. Examination of collagen alignment revealed decreased collagen alignment in immobilized mice as well as decreased MPC cell number and cell spread, suggesting a deficiency in migration within the ECM. This in vivo finding was recapitulated in vitro using aligned and nonaligned collagen type I functionalized electrospun fibrous matrices, given that the Achilles tendon has high collagen I content. Similar to in vivo findings, MPCs grown on nonaligned fibers had increased adipogenic differentiation compared with aligned fibers. scATACand scRNA-sequencing analysis of mice immobilized or allowed to ambulate after injury (mobilized), confirmed that MPCs from immobile mice had a trajectory and open chromatin regions in genes associated with adipogenesis while trajectories and chromatin accessibility in MPCs from mobile mice was associated with osteogenesis. Together, these data suggest that joint immobilization alters MPC fate, protecting from $\mathrm{HO}$ formation and promoting adipocyte differentiation.

\section{Results}

MPC characterization of the extremity injury site. To examine the phenotype of MPCs that aberrantly differentiate into bone after severe extremity injury, we performed unbiased single-cell sequencing analysis of the whole-tissue homogenate harvested from the $\mathrm{HO}$ anlagen after a $30 \%$ total body surface area burn and concurrent tenotomy $(\mathrm{B} / \mathrm{T})$ injury (Figure $1 \mathrm{~A})$. All cells from the injury site were sequenced to ensure capture of all mesenchymal cell phenotypes present. To our knowledge, MPC subpopulations from the HO anlagen have not been previously defined. Samples were collected at day 0 (uninjured), 3, 7, and 21 after injury, for a total of 13,362 cells after quality filtering. Unsupervised clustering applied to the canonical correlation analysis of the 4 data sets yielded 16 transcriptionally unique cell clusters identifiable at the injury site (Figure 1B). We identified characteristic profiles attributable to known cell types for MPCs, endothelial cells, pericyte/ smooth muscle cells, macrophages, granulocytes, dendritic cells, neural cells, lymphocytes, and neuromuscular junction cells (Supplemental Figure 1A; supplemental material available online with this article; https://doi.org/10.1172/JCI136142DS1). One small cluster (95 cells) remained uncharacterized. Three clusters (clusters 0, 6, and 8) were classified as MPCs, defined by expression of both Pdgfra and Prrx1. Pathway analysis comparing differentially expressed genes from day 7 in the 3 MPC clusters after injury revealed pathways associated with focal and cell adhesion, and ECM-receptor interactions (Table 1). Further, gene ontogeny (GO) analysis revealed terms associated with ECM organization, cell adhesion and migration, response to stimuli, and cell proliferation
(Table 2). Focal adhesion kinase (FAK) is known to be important in regulating downstream signaling events after cells form focal adhesions with the ECM (20). Other mechanotransduction signals are also important, such as the transcriptional coactivators YAP1 and TAZ, which have been shown to be important mediators in response to mechanical stimuli such as cell spread (21-23). MPC cluster 6 appears to be less differentiated and is comprised mostly of cells from day 0 , whereas clusters 0 and 8 appear to be more differentiated, having higher expression of genes associated with differentiation (Supplemental Figure 1B). Analysis of expression of those genes encoding FAK (Ptk2), YAP1 (Yap1), and TAZ (Wwtr 1 ) in clusters 0,6 , and 8 demonstrated that at day 7 and day 21, time points when the MPCs will be differentiating for tissue repair, Ptk2, Yap1, and Wwtr1 had high fold changes compared with day 0 , particularly in clusters 0 and 8 (Figure 1C). Our model of injury and repair suggests a role of FAK and YAP/TAZ signaling in MPC cluster differentiation.

To assess the hypothetical developmental stage of differentiation in the MPC clusters, we performed a trajectory analysis of clusters 0,6 , and 8 using Monocle (Figure 1D). The analysis revealed that MPCs followed a trajectory that resulted in branches with characteristics of tenogenic, chondrogenic, and osteogenic fates (Figure 1D). Of note, while all 3 clusters were identified as MPCs based on the expression of previously identified markers, there was heterogeneity seen within and between the clusters and based on the trajectory analysis. This heterogeneity is more diverse than previously defined by lineage tracing mouse studies $(12-16,18)$.

Given our unbiased transcriptomic identification of Ptk2, Yap1, and Wwtr1 expression in $\mathrm{HO}$, we next moved to validate these findings by performing immunofluorescence staining for FAK, pFAK, nuclear TAZ, and PDGFR 7 days after burn/tenotomy (Figure 2A and Supplemental Figure 1C). The region surrounding the Achilles tendon where $\mathrm{HO}$ usually forms was highly enriched with PDGFR $\alpha^{+}$ MPCs. Nearly $80 \%$ of PDGFR $\alpha^{+}$MPCs were positive for pFAK staining (Figure 2A), whereas only $20 \%$ of PDGFR $\alpha^{+}$MPCs colocalized with pFAK in uninjured samples. Further, to analyze active TAZ signaling we performed immunofluorescence staining of TAZ and found around a 4 -fold increase in nuclear translocated TAZ in MPCs 7 days after burn/tenotomy $(24,25)$ compared with the analogous noninjured regions (Figure 2A). Significant differences in FAK and TAZ signaling in MPCs were still seen 3 weeks after B/T (Figure 2B).

To determine whether these findings translate into the formation of trauma-induced HO in human tissue, we next analyzed samples of early human traumatic HO for the expression of FAK/pFAK costained with the MPC marker PDGFR $\alpha$. There was robust FAK and pFAK staining specifically in MPCs (PDGFR $\alpha^{+}$cells) by immunohistochemistry. Comparatively, there was little or no staining in unaffected healthy bone (Figure 2C). Together, these data suggest that mechanotransductive signaling through FAK, YAP1, and TAZ is increased during the development of trauma-induced HO.

$M P C$-targeted deletion of FAK and TAZ alters aberrant cell fate. To determine whether the increased mechanotransduction signaling we see at the injury site is important for the aberrant cell fate seen during our B/T model, we performed B/T and analyzed HO formation in $\mathrm{Pr} x-\mathrm{Cr}^{+} \mathrm{Fak}^{\mathrm{fl} / \mathrm{ll}}$ mice, where FAK is specifically knocked out in MPCs, and littermate controls. Our group has previously demonstrated using Prx-cre reporter mice (Prx-cre R26mtmg/+) that over 
A

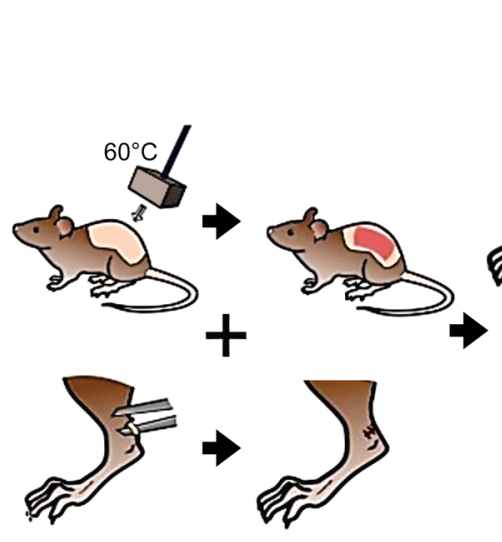

B

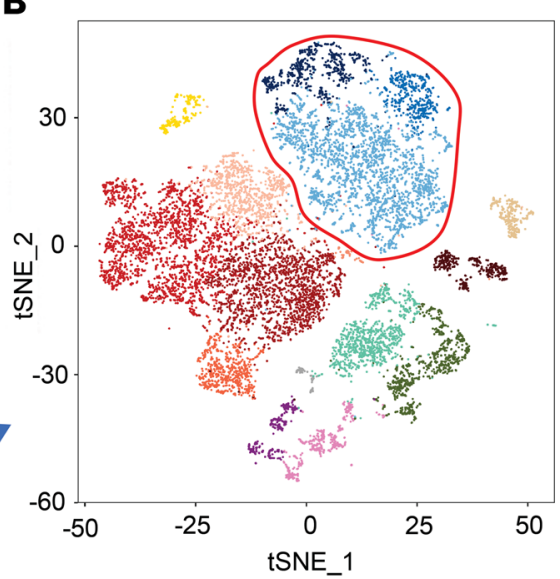

Pdgfra

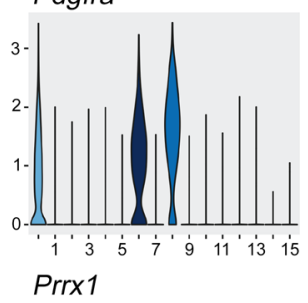

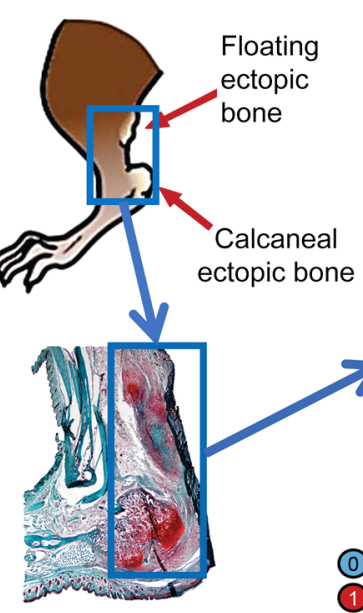

(2) Mesenchymal (4) Macrophage (8) Mesenchymal

(1) Macrophage (5) Endothelial (9 Pericyte/Smooth Muscle (13) Neural

(2) Macrophage (6) Mesenchymal (1) Lymphocyte

(3) Endothelial (I) Dendritic cell (1) Pericyte/Smooth Muscle (1)

(14) Undefined

(1) Granulocyte
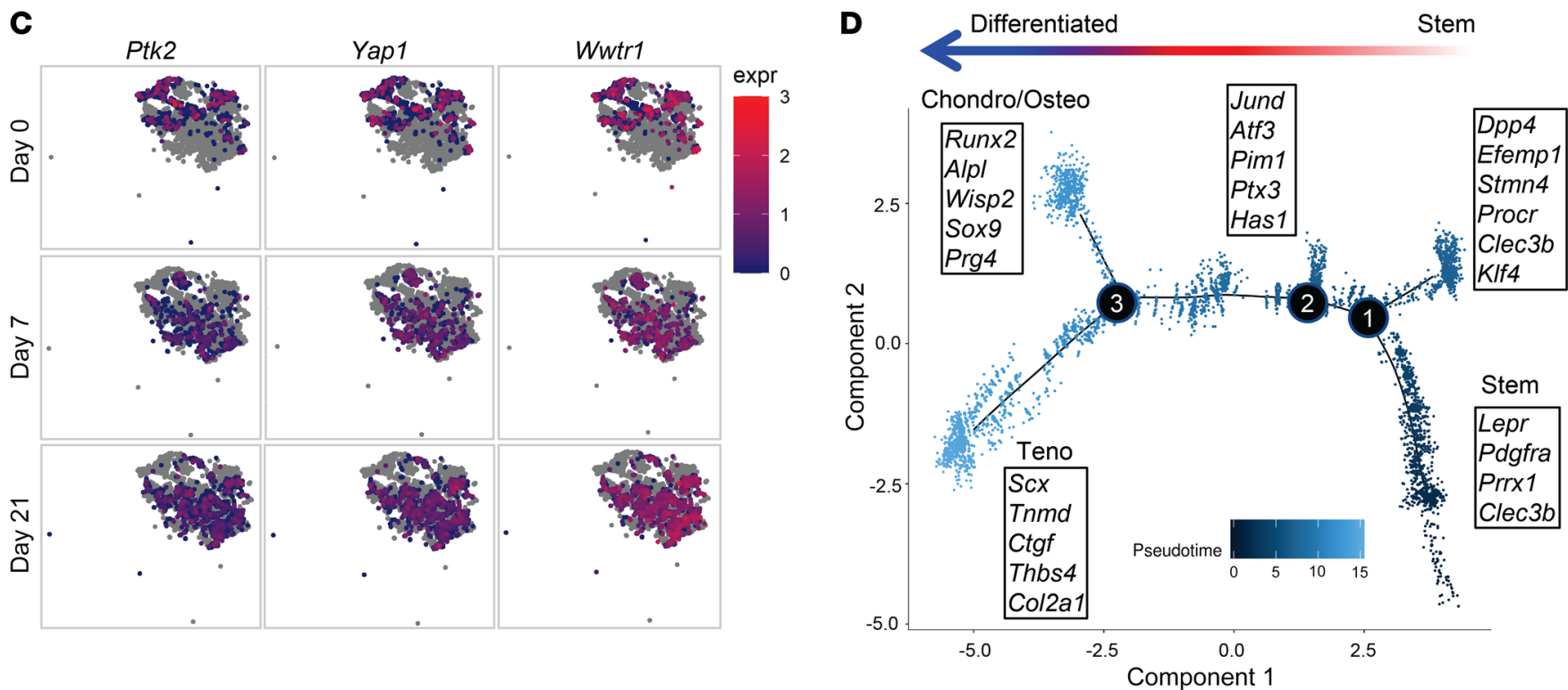

Figure 1. MPCs at the extremity injury site demonstrate increased mechanotransductive genes before aberrant cell fate change. (A) Schematic of burn/ tenotomy (BT) injury model denoting where the cells were harvested (blue box). (B) Canonical correlation analysis of the HO site defines 16 clusters, including 3 MPC subsets based on expression of Pdgfra, Prrx1, and Clec3b. (C) Feature plot of the MPC clusters displaying expression of Ptk2, Wwtr1, and Yap1 across the different time points of the canonical analysis. (D) Trajectory analysis of gene expression changes in cells across pseudotime.

$95 \%$ of cells at the tendon transection site and developing $\mathrm{HO}$ anlagen are marked by Prx-Cre (GFP $)$ (12). Because cells expressing Prrx1 (gene encoding Prx1) are important for normal development, we analyzed tibial cortical thickness to make sure that $\mathrm{Prx}-\mathrm{Cre}^{+} \mathrm{Fak} \mathrm{k}^{\mathrm{f} / \mathrm{I}}$ did not have inherent differences that might compound the results in our study. We found that both $\operatorname{Prx}-\mathrm{Cr}^{+} \mathrm{Fak}^{\mathrm{A} / \mathrm{l}}$ and littermate controls had similar tibial cortical thickness; however, the $\mathrm{Pr} x-\mathrm{Cre}^{+}$ $\mathrm{Fak}^{\mathrm{B} / \mathrm{l}} \mathrm{h}$ had decreased tibial length (Supplemental Figure 2, A and B). After B/T, we discovered over $70 \%$ less distal, non-bone associated HO in $\mathrm{Prx}-\mathrm{Cre}^{+} \mathrm{Fak}^{\mathrm{t} / \mathrm{l}}$ mice compared with littermate controls (Figure 3A). It has been shown that inhibiting FAK does not affect the formation of FAs; however, downstream Rac 1-driven cellular processes such as cell migration and proliferation are completely inhibited (20), therefore, we next sought to analyze the number and MPC spread in $\mathrm{Pr} x-\mathrm{Cre}^{+} \mathrm{Fak}^{\mathrm{n} / \mathrm{l} / \mathrm{l}}$ mice. We found no differences in the ability of MPCs to spread or migrate into the $\mathrm{HO}$ site in the $\mathrm{Prx}-\mathrm{Cre}^{+}$ $\mathrm{Fak}^{\mathrm{n} / \mathrm{fl}}$ mice, as there were similar numbers of MPCs at the future HO site at day 7, as well as a similar MPC cell spread and area at days 7 and 21 (Figure 3B). However, 3 weeks after injury, there were significantly more MPCs at the HO site in WT controls compared with the $\mathrm{Pr} x-\mathrm{Cr}^{+} \mathrm{Fak} k^{\mathrm{n} / \mathrm{l}}$ mice, suggesting a defect in cellular proliferation in $\mathrm{Prx}$ - $\mathrm{Cre}^{+} \mathrm{Fak}^{\mathrm{n} / \mathrm{l}}$ MPCs.

Given the results in our $\operatorname{Pr} x-\mathrm{Cre}^{+} \mathrm{Fak}^{\mathrm{n} / \mathrm{l} l}$ mice with severe trauma, we considered the possibility that pharmacologic inhibition 


\section{Table 1. Mechanotransductive pathways of differentially expressed genes from day 0 to day 7}

$\begin{array}{lcc}\text { Cluster } & \text { Pathway } & \boldsymbol{P} \\ 0 & \text { ECM-receptor interaction } & 3.09807 \times 10^{-6} \\ 0 & \text { Focal adhesion } & 0.000195337 \\ 6 & \text { ECM-receptor interaction } & 0.045332 \\ 8 & \text { ECM-receptor interaction } & 0.00067118 \\ 8 & \text { Focal adhesion } & 0.012 \\ 8 & \text { Cell adhesion molecules } & 0.024\end{array}$

of FAK might mitigate posttraumatic $\mathrm{HO}$ as a future translational strategy. To test the effect of pharmacologically inhibiting FAK in vivo on the formation of $\mathrm{HO}$, we treated mice daily for 3 weeks after B/T by SQ injection of FAK inhibitor PF573228 (26). We used PF573228 because it has been shown to be highly specific at inhibiting FAK kinase activity without affecting a related kinase, Pyk2 (27). This is important because activation of Pyk2 is not related to mechanotransduction, but instead to cytokines, growth factors, and increases in cytosolic $\mathrm{Ca}^{+}$. Final $\mathrm{HO}$ volume, as assessed by microCT, demonstrated that PF573228 significantly decreased $\mathrm{HO}$ volume to nearly half the level of the vehicle-treated control (Figure 3C), consistent with genetic Ptk2 deletion.

Further, to demonstrate the importance of TAZ in the formation of trauma-induced $\mathrm{HO}$ we induced the deletion of YAP1 and TAZ by treating Hoxa11CreER Yap ${ }^{\mathrm{A} / \mathrm{l}} \mathrm{Taz}^{\mathrm{A} / \mathrm{fl}}$ or littermate control mice with tamoxifen and performed B/T. Hoxa11 is an embryonic patterning gene expressed specifically in the MPCs of the zeugopod (28) (tibia/fibula, radius/ulna). Using lineage tracing Hoxa11CreER R26 $6^{\text {TdTomato }}$ reporter mice, we have shown in our laboratory that these MPCs become HO in our B/T model (Supplemental Figure 2C). We chose to use these mice, as Yap1 and Wwtr1 would be deleted only in the MPCs of the zeugopod, the site of our extremity injury, to avoid any confounding effects of a global deletion (29-32). In these mice, Yap1 and Wwtr1 (the gene encoding Taz) are knocked out in MPCs of the zeugopod (radius/ulna and tibia/ fibula). Similar to FAK deletion, B/T injury in the Hoxa11CreER Yap ${ }^{\mathrm{n} / \mathrm{l}} \mathrm{Taz}^{\mathrm{A} / \mathrm{I}}$ mice resulted in significantly decreased $\mathrm{HO}$ formation as compared with the WT littermate controls (Figure 3D).

Together, these data suggest that mechanotransductive signaling in MPCs, through FAK or YAP/TAZ, is important for the formation of HO. Though promising, pharmacologic inhibitors to these molecules often have off-target effects. Given the ankle is an anatomical site of high mechanical stimuli and stress properties that induce mechanotransductive signaling, we sought to determine whether immobilizing the joint after injury would provide a nonpharmacologic approach to alter mechanotransduction, and thus mitigate $\mathrm{HO}$ in our model.

Joint immobilization decreases mechanotransduction signaling and HO formation. A large body of literature supports cellextrinsic forces altering MPC osteogenic signaling and differentiation (33-38). We leveraged these previous in vitro findings and analyzed the formation of bone in vivo after $\mathrm{B} / \mathrm{T}$ along with joint immobilization (Supplemental Figure 3A) of the ankle (described in ref. 39). Although limb suspension models exist, these models still allow the ankle to move despite mitigating ambulation (40). The role of joint mobilization on HO development remains controversial as some studies have shown forced mobilization to prevent $\mathrm{HO}$ whereas others have shown forced mobilization to be detrimental (41-44). In order to address this, we compared forced mobilization, passive range of motion, and complete immobilization. A cohort of mice forced to be on a treadmill daily for 1 hour did not form any less HO than those mice allowed to ambulate normally. Similarly, passive range of motion exercises (from $25^{\circ}-160^{\circ}$ once a day), which is commonly performed for patients after surgery or a burn injury, also did not decrease posttraumatic HO. However, complete immobilization of the joint at risk for HO entirely mitigated bone formation (Figure 4A). Therefore, for the remaining studies we used those mice allowed to ambulate normally, or mobile mice, compared with immobilized mice.

Next, we analyzed mechanotransductive signaling by immunostaining for active FAK (pFAK) and the nuclear translocation of transcriptional coactivator TAZ, representing activated signaling, at the $\mathrm{HO}$ anlagen of mobile and immobile mice. We chose to analyze TAZ, as it has been shown to regulate MPC osteogenesis and adipogenesis (45). Our data revealed that immobilized mice had significantly decreased levels of total $\mathrm{FAK}^{+}$and $\mathrm{pFAK}^{+}$, PDGFR $\alpha^{+}$

Table 2. Gene ontogeny analysis, days $\mathbf{0}$ and $\mathbf{7}$

\begin{tabular}{|c|c|c|c|c|c|}
\hline Cluster & $\begin{array}{c}\text { Gene Ontology } \\
\text { ID }\end{array}$ & Name & $\begin{array}{c}\text { Differentially } \\
\text { expressed genes }\end{array}$ & $\begin{array}{l}\text { All } \\
\text { genes }\end{array}$ & $P$ \\
\hline \multicolumn{6}{|l|}{ Cluster 0} \\
\hline & G0:0043062 & Extracellular structure organization & 34 & 143 & $1.90 \times 10^{-12}$ \\
\hline & CO:0030198 & Extracellular matrix organization & 32 & 131 & $3.40 \times 10^{-12}$ \\
\hline & G0:0022610 & Biological adhesion & 57 & 433 & $7.81 \times 10^{-11}$ \\
\hline & G0:0050896 & Response to stimulus & 153 & 2164 & $7.81 \times 10^{-11}$ \\
\hline & GO:0007155 & Cell adhesion & 56 & 432 & $1.43 \times 10^{-10}$ \\
\hline \multicolumn{6}{|l|}{ Cluster 6} \\
\hline & CO:0042127 & Regulation of cell proliferation & 40 & 495 & $1.92 \times 10^{-7}$ \\
\hline & G0:0050896 & Response to stimulus & 85 & 1874 & $2.19 \times 10^{-7}$ \\
\hline & G0:0016477 & Cell migration & 38 & 467 & $5.76 \times 10^{-7}$ \\
\hline & G0:0007166 & Cell surface receptor signaling pathway & 46 & 661 & $6.91 \times 10^{-7}$ \\
\hline & G0:0008283 & Cell proliferation & 42 & 580 & $1.8437 \times 10^{-6}$ \\
\hline \multicolumn{6}{|l|}{ Cluster 8} \\
\hline & G0:0030198 & Extracellular matrix organization & 19 & 140 & $2.53 \times 10^{-9}$ \\
\hline & GO:0030155 & Regulation of cell adhesion & 22 & 261 & $3.78 \times 10^{-7}$ \\
\hline & GO:0007155 & Cell adhesion & 28 & 439 & $4.71 \times 10^{-7}$ \\
\hline & G0:0022610 & Biological adhesion & 28 & 442 & $4.71 \times 10^{-7}$ \\
\hline & G0:0031589 & Cell-substrate adhesion & 16 & 149 & $2.0377 \times 10^{-6}$ \\
\hline & GO:0048870 & Cell motility & 30 & 579 & $6.6267 \times 10^{-6}$ \\
\hline
\end{tabular}


A

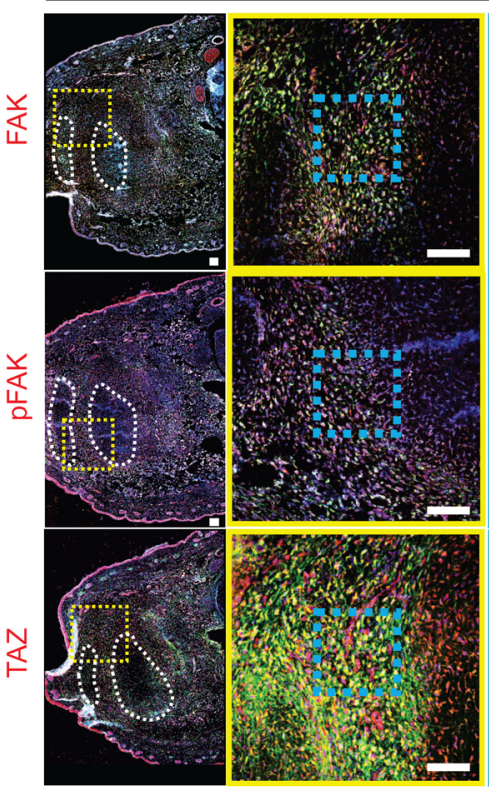

Hoechst

B

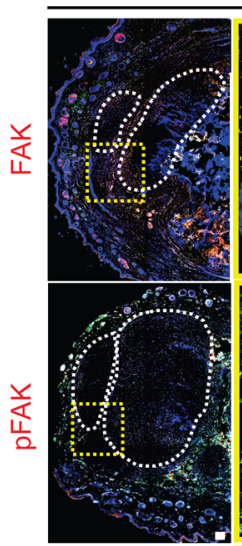

3 Week burn/tenotomy
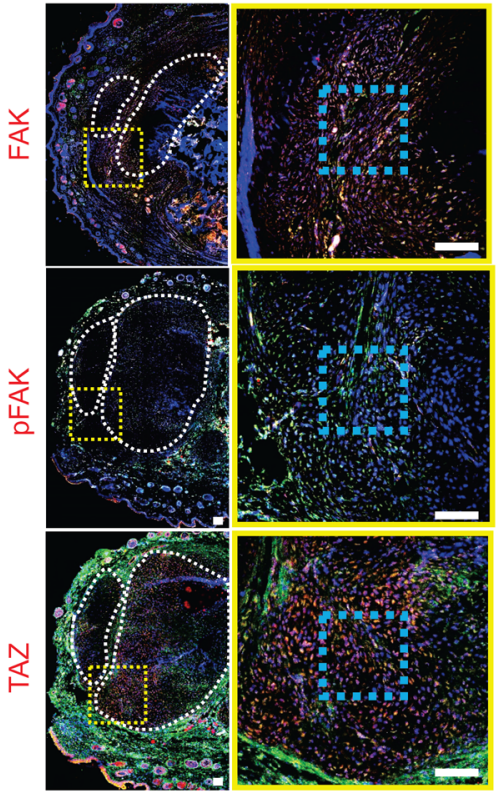

Hoechst
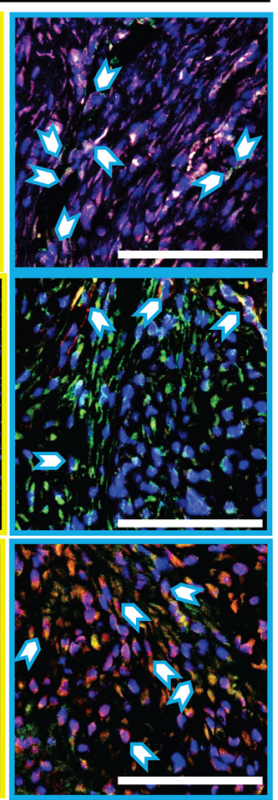

Uninjured bone

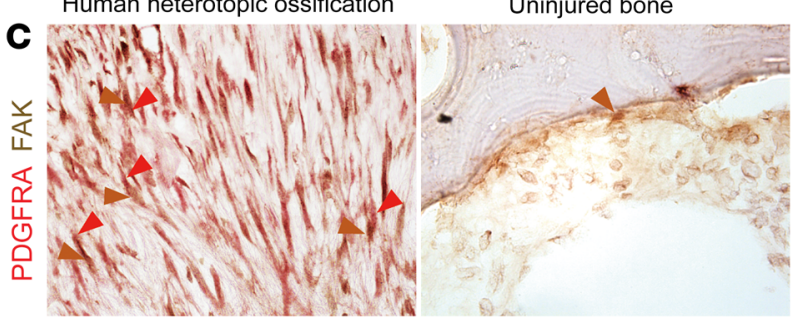

PDGFRa

Uninjured
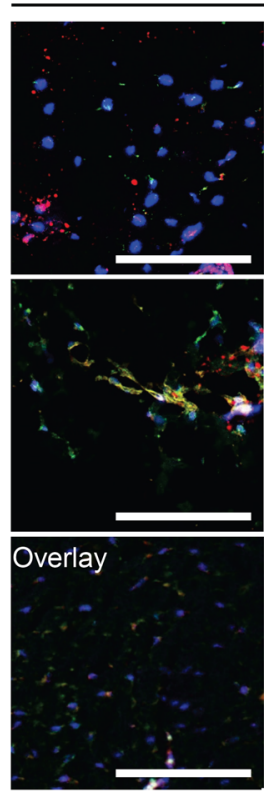

PDGFRo
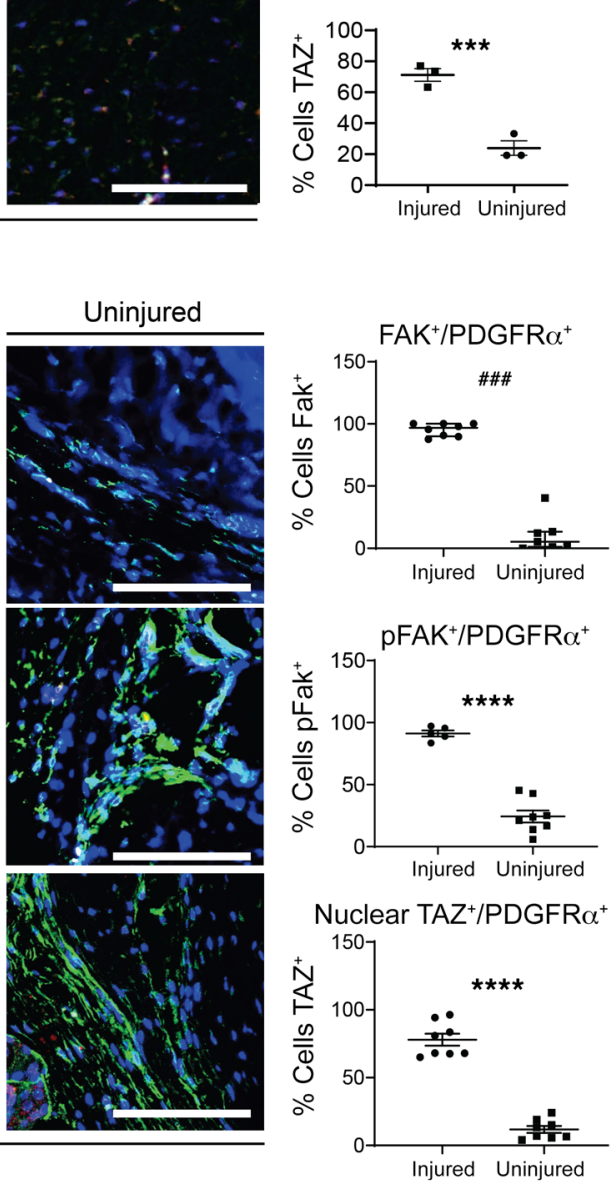

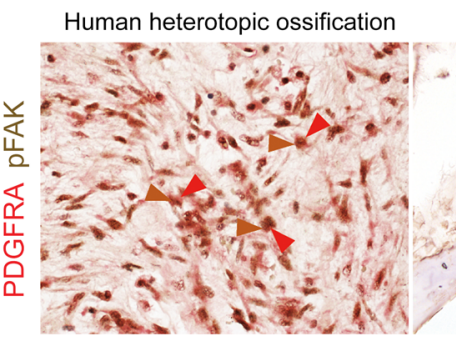

Uninjured bone 
Figure 2. MPCs at the extremity injury site demonstrate increased mechanotransductive signaling before aberrant cell fate change. (A) Confocal microscopy images of injured and uninjured mouse hind limbs immunologically stained with anti-PDGFR $\alpha$ and anti-FAK, anti-pFAK, or anti-TAZ after 1 week BT injury compared with uninjured control. Nuclei are stained with Hoechst 33342 . Tilescan images (left) of $\mathrm{HO}$ anlagen with tendon encircled by white dotted outline and red dotted square showing $\times 20$ image (middle). Image overlay at $\times 20$ magnification with individual channels (right). Blue-dotted square shows $\times 63$ magnification. Image overlay at $\times 63$ magnification with individual channels (right). Image overlay at $\times 20$ magnification of uninjured mouse hind limb with individual channels (right). Quantification of $\times 63$ magnification comparing number of PDGFR $\alpha^{+}$ cells expressing FAK, pFAK, and nuclear TAZ, respectively in injured and uninjured hind limbs by independent samples $t$ test $\left(n=3 /\right.$ group, ${ }^{* *} P$ $<0.001$ ). (B) FAK, pFAK, and TAZ immunofluorescent stains at 3 weeks postinjury ( $n=3-4$ /group) of injured and uninjured mouse hind limbs immunologically stained with anti-PDGFR $\alpha$ and anti-FAK, anti-pFAK, or anti-TAZ. Scale bars: $100 \mu \mathrm{m}$. ${ }^{\# \# P}<0.001,{ }^{* * * *} P<0.0001$. (C) Immunohistochemical stains of FAK, pFAK, and PDGFR $\alpha$ of human uninjured bone and HO. Original magnifications, left to right: $\times 40, \times 20, \times 40$, and $\times 20$.

MPCs 7 days after B/T (Figure 4B and Supplemental Figure 3B). Further, we found a decrease in nuclear TAZ (Figure 4B), which was consistent with previous studies analyzing tissue fibrosis (46). To confirm decreased TAZ signaling with immobilization we also stained for the downstream target of TAZ transcriptional coactivator, connective tissue growth factor (CTGF). We found decreased CTGF in $\mathrm{B} / \mathrm{T}$ with immobilization, further confirming decreased active TAZ signaling (Figure 4B). Of interest, in our immunofluorescence images, we noted that immobilized mice had large pockets of empty space where no cells appeared to be present. These black areas appeared spherical in nature; therefore, we hypothesized that they might be adipocytes. To test the difference in adipocytes present in immobile and mobile mice, we performed Oil Red O (ORO) stain and immunofluorescence staining for perilipin. We found that in immobilized mice, there was significantly more ORO staining (Figure 5A) and perilipin stain (Figure 5B) present surrounding the Achilles tendon, suggesting increased adipogenesis in immobilized mice. These data suggest that immobilization of the injury site potentially decreases osteogenesis while increasing adipogenesis. Given the extensive literature on the effect of substrate stiffness on MPC differentiation toward bone $(21,47)$, we hypothesized that with immobilization, the extracellular matrix in the region where HO forms would be less stiff than in mobile mice.

Immobilization alters the extracellular environment affecting MPCs. To test whether joint immobilization results in decreased tissue stiffness that might drive MPC differentiation toward adipocytes instead of osteocytes, we performed atomic force microscopy (AFM) on one-week B/T samples. Unexpectedly, AFM demonstrated increased tissue stiffness at the $\mathrm{HO}$ anlagen in immobilized mice compared with mobile counterparts (Figure 6A). In addition to our AFM results, we also used dynamic mechanical analysis (DMA) (48) on the HO anlagen 14 days after $\mathrm{B} / \mathrm{T}$, which also confirmed an increased stiffness of the $\mathrm{HO}$ site in immobile compared with mobile mice (Supplemental Figure 3C).

Having found increased stiffness in immobilized mice, this suggested that aberrant osteo-differentiation was not likely due to the effect of tissue stiffness on MPCs. To determine whether the type of collagen present contributed to the differences in stiffness we found in our immobilized mice, we analyzed type 1,2 , and 3 collagens by immunofluorescence. We found similar amounts of all types of collagen present in mobile and immobile mice (Supplemental Figure 3D).

We next employed second-harmonic generation (SHG) microscopy to visualize ECM collagen of the HO anlagen after B/T (Figure 6B and Supplemental Figure 3E). We observed qualitative and quantitative differences between mobile and immobilized limbs. Immobilized mice reproducibly had denser, more disorganized (entropic) distributions of collagen fibrils, compared with more aligned fibers in the mobile mice. Because it has been shown that inhibiting cell spreading can result in decreased focal adhesion stability, decreased YAP/TAZ activity, and altered differentiation toward adipogenesis $(21,49)$, we wanted to assess cell spreading in mobile and immobilized mice. We examined cell aspect ratio as well as cell spread area and found that MPCs at the injury site in immobilized $\mathrm{B} / \mathrm{T}$ mice were more rounded and less stretched than those present in mobile mice (Figure 6C). Additionally, Western blot analysis of lysates prepared from the $\mathrm{HO}$ anlagen in mobile and immobile mice revealed that there was decreased prochondrogenic TGF- $\beta 1$ signaling (pSMAD2) and an increase in the adipogenic-associated transcription factor PPAR $\gamma$ in immobile mice compared with mobile mice (Figure 6D).

To determine whether ECM fiber alignment in particular influenced MPC fate as we saw in vivo with immobilization, we performed in vitro studies using functionalized engineered synthetic extracellular matrices with control over fiber alignment. MPCs harvested from the injury site of mobilized B/T mice 1 week after injury were plated on either aligned or nonaligned electrospun polymer fibers functionalized with type I collagen, corresponding to the collagen organization noted in mouse tissues by SHG imaging. We chose to use type I collagen given its presence after $\mathrm{B} / \mathrm{T}$ and because the Achilles tendon possesses one of the highest concentrations of collagen type 1 in the body. Similar to what we saw in our immobilized mice in vivo, immunocytochemistry staining (ICC) demonstrated that cells plated on nonaligned fibers had a more rounded appearance, with decreased cell spreading as quantified by cell area (Figure 6E). Quantification of focal adhesions on aligned and nonaligned fibers demonstrated a decrease in the number of focal adhesions formed on nonaligned fibers (Figure $6 \mathrm{~F}$ ). However, ICC staining for activated FAK (pFAK) demonstrated no difference in the level of FAK with respect to fiber alignment (Figure 6G). Further, we noted decreased MPC migration (speed and distance; Figure 6H) on nonaligned fibers, remaining immobile and lacking polarity, whereas on the aligned fibers, cells extended and migrated along the direction of the fibers (Supplemental Video 1). Next, we assessed in vivo the number of MPCs 1 week after injury in mobile and immobile mice. Unlike the $\operatorname{Pr} x-\mathrm{Cre}^{+} \mathrm{FAK}^{\mathrm{t} / \mathrm{fl}}$ mice in vivo, immobilized mice had decreased numbers of MPCs present 7 days after injury at the $\mathrm{HO}$ anlagen (Figure 6I). Further, similar to the decreased TAZ nuclear translocation we saw with immobilization (Figure 3B), MPCs plated on nonaligned fibers had significantly decreased amounts of nuclear translocated TAZ compared with aligned (Figure 6J). Because it has been shown that activated TAZ signaling impacts cellular 
A

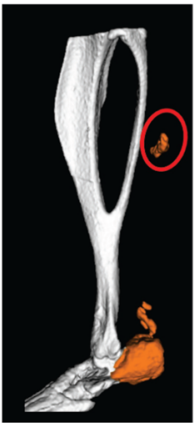

PrxCre FAK ${ }^{\text {wtf }}$

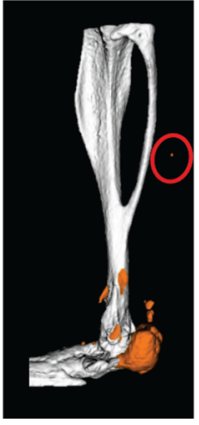

PrxCre FAK
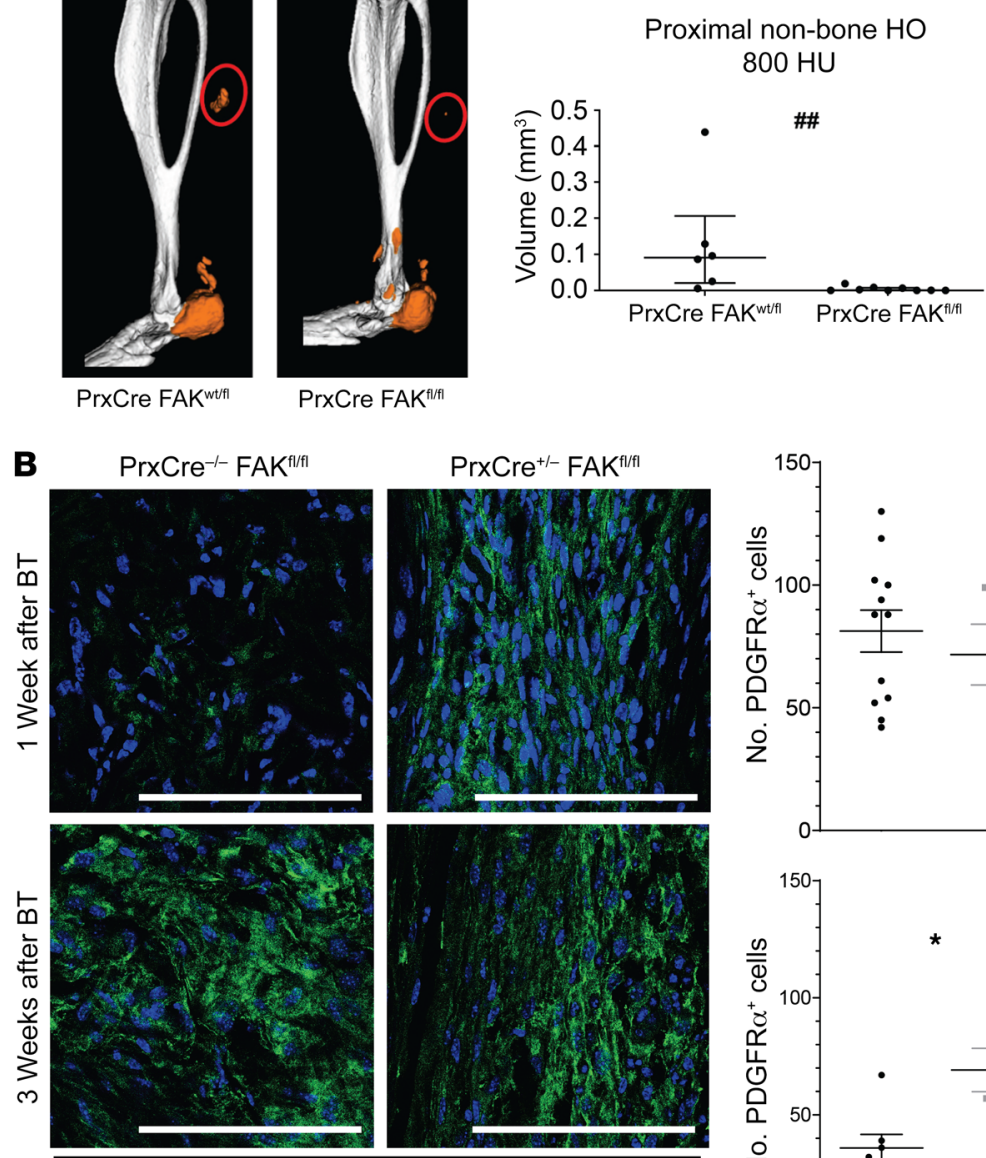

Hoechst PDGFR
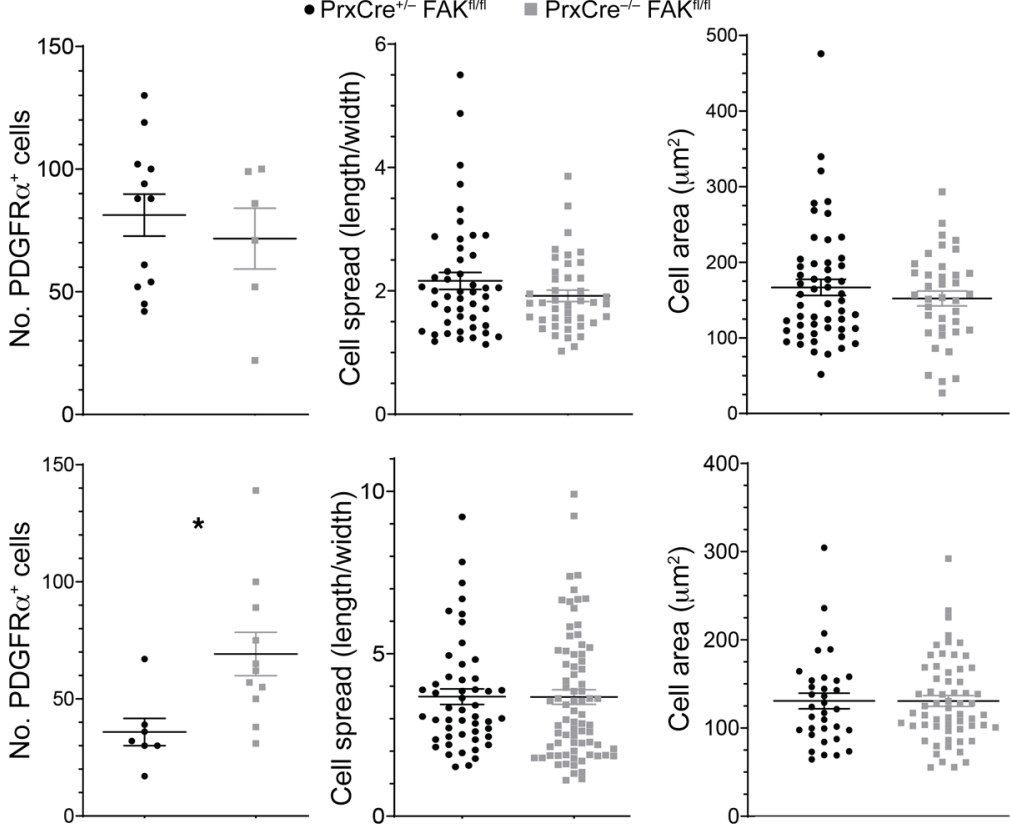

C

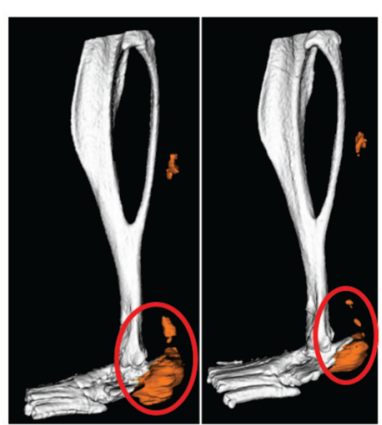

Vehicle ctrl

FAKi

D

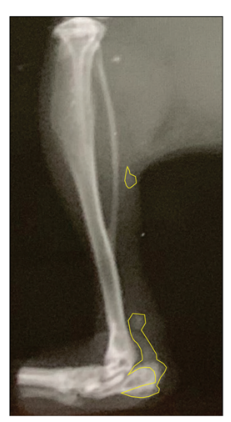

Hoxa11CreER $R^{T 2}$ YAP $P^{\text {f/ff }} T A Z^{1 / / f}$

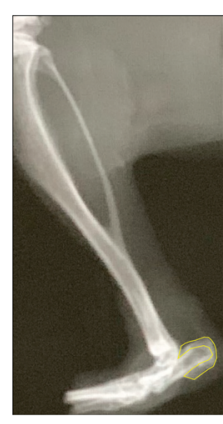

Hoxa11CreER ${ }^{T 2}$ $Y A P^{f / f f l} T A Z^{f / / f l}$
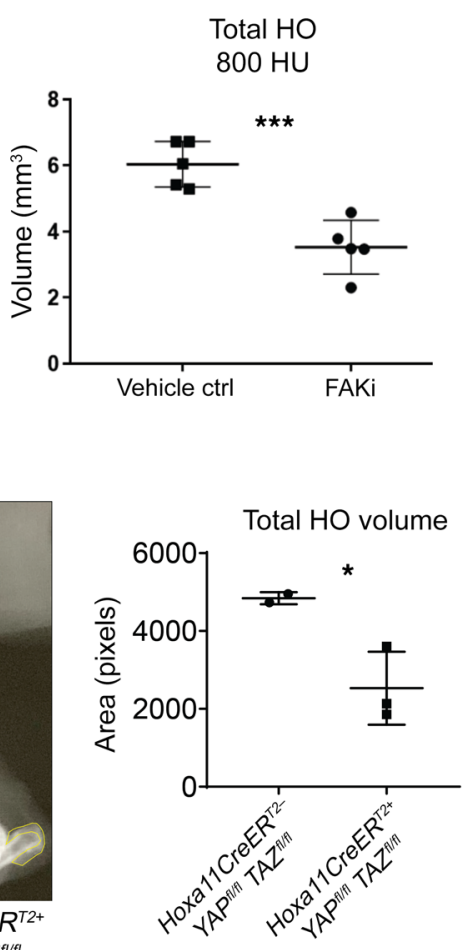

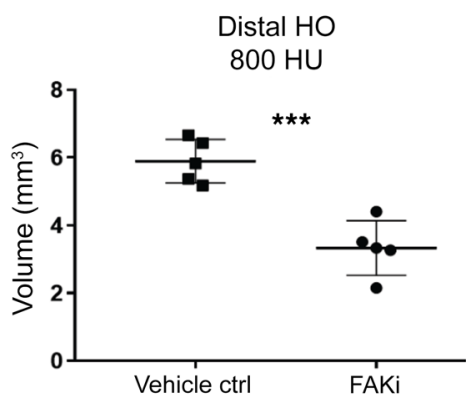

Figure 3. FAK deletion or inhibition reduces heterotopic bone. (A) Deletion of FAK (Ptk2) within the Prx lineage reduces proximal non-bone $\mathrm{HO}$ compared with mouse with single wt allele in Prx lineage by $\mu \mathrm{CT}$ imaging at 800 Hounsfield units (HU) ${ }^{\# \# ~} P<0.01, n=$ 6-9/group, Mann-Whitney $U$ test). (B) Confocal microscopy images of $\mathrm{Prx}-\mathrm{Cr} \mathrm{r}^{-}$and $\mathrm{Prx}-\mathrm{Cre}^{+}$deletion of FAK probed with indicated antibodies at 1 and 3 week time points after injury (left) quantified PDGFR $\alpha$ cell number, cell spread, and cell area $\left({ }^{*} P<0.05, n=2-4\right.$ / group, $n=2-4$ roi/mouse, $n>15$ cells/image, Student's $t$ test). Scale bars: $100 \mu \mathrm{m}$. (C) FAK inhibitor (FAKi) PF573228 treated mice showed reduced total and distal $\mathrm{HO}$ at $800 \mathrm{HU} 9$ weeks after injury (*** $P<0.001, n=5$ /group, Student's $t$ test). (D) Inducible conditional deletion of YAP and TAZ coactivators within Hoxa11expressing cells causes $50 \%$ reduction in ectopic bone formation Student's $t$ test ( ${ }^{*} P<0.05, n=2-3$ mice/group). 
A

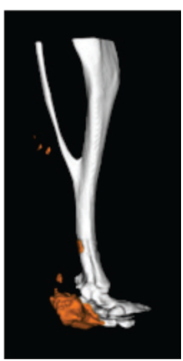

Passive ROM

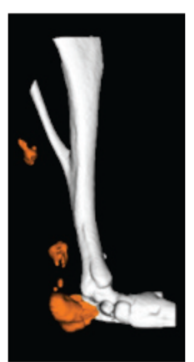

Treadmill run

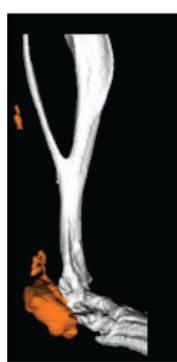

Mobilized

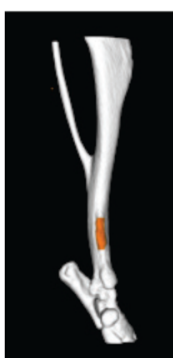

Immobilized

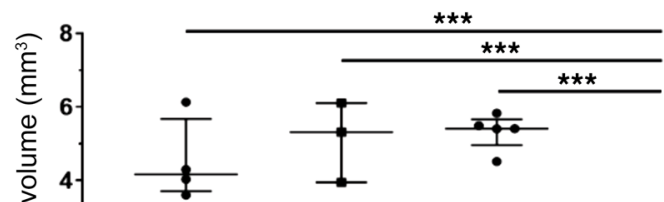

1 Week mobilized
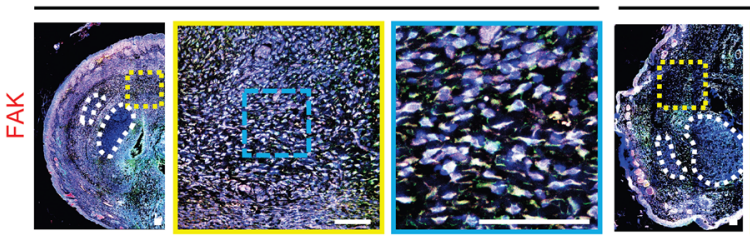

1 Week immobilized
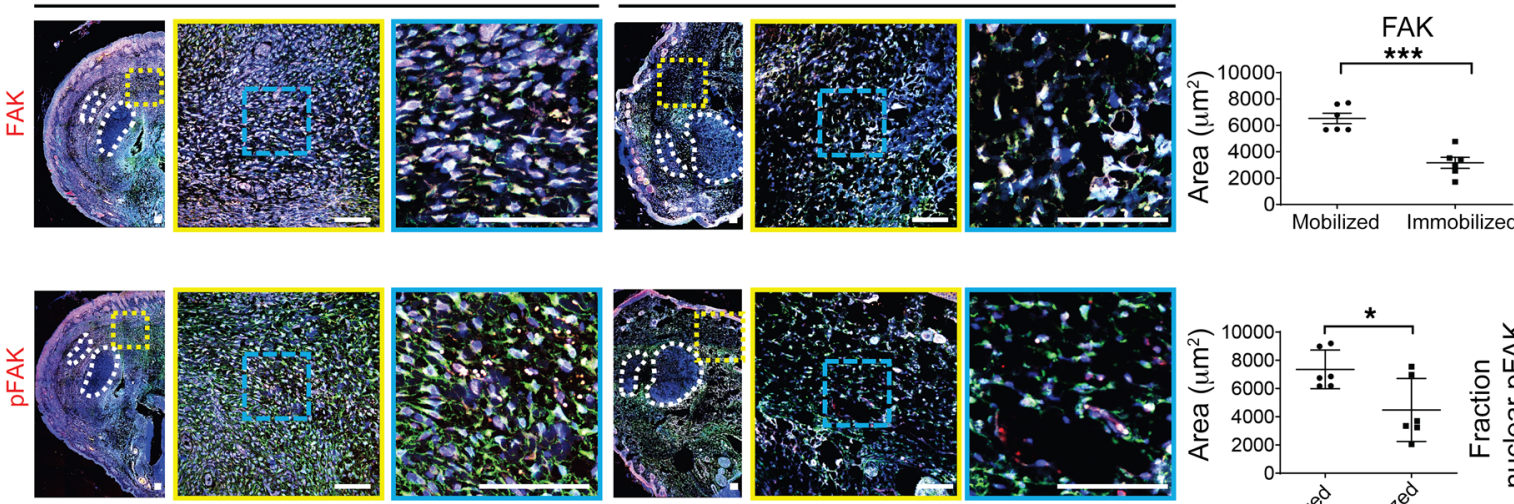

Passive

ROM

Treadmill

run
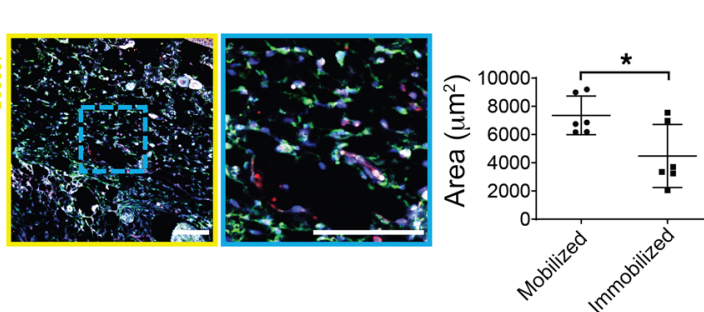

Proportion nuclear pFAK signaling
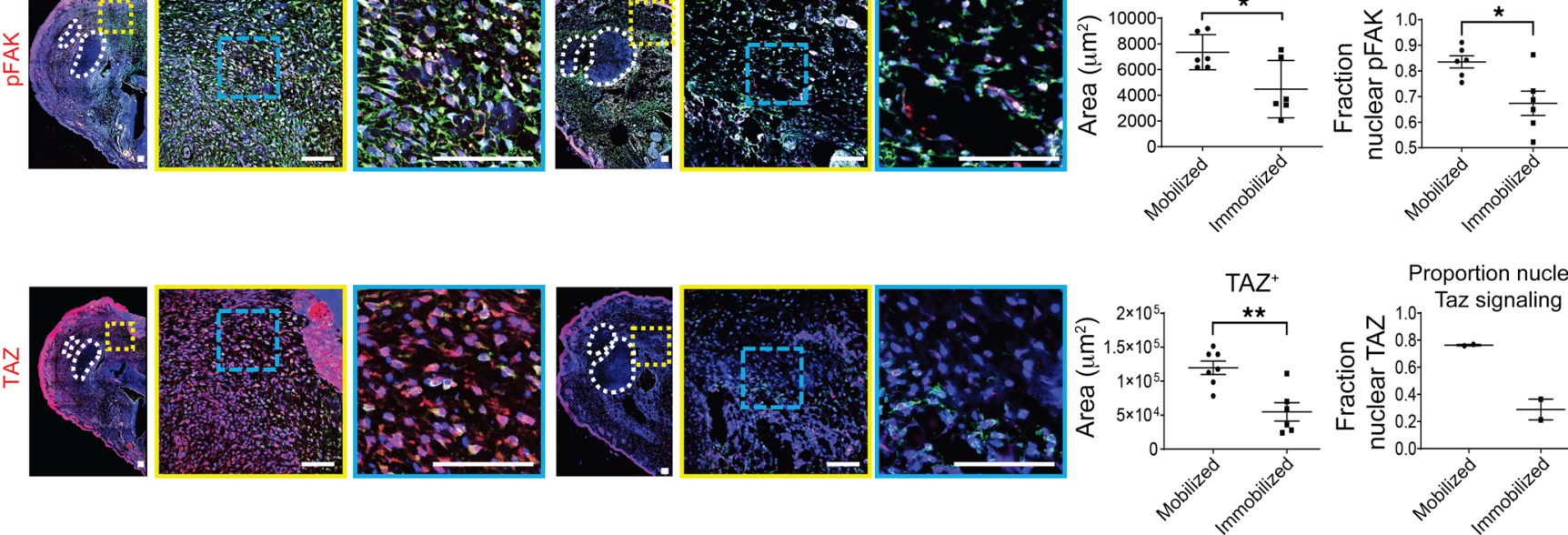

Proportion nuclear

Proportion $\mathrm{CTGF}^{+} \mathrm{PDGFR}^{+}$cells/ total PDGFR $\alpha^{+}$cells
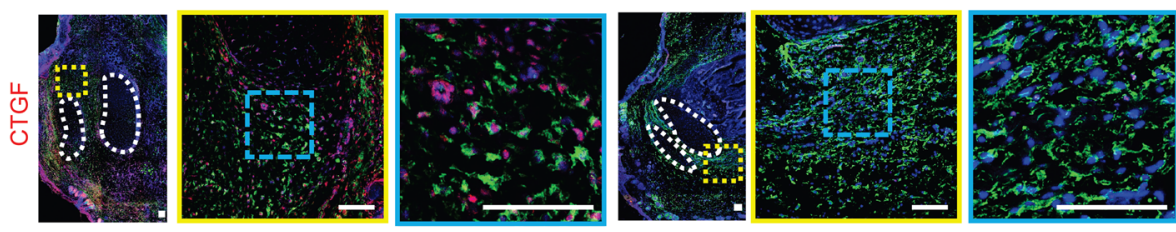

Hoechst PDGFR

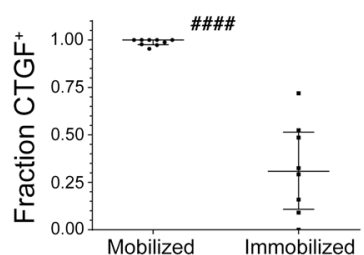

Figure 4. Hind limb immobilization reduced HO formation and alters cell fate. (A) $\mu$ CT imaging of passive range of motion, forced mobilization, normal ambulation, and complete immobilization groups 9 weeks after injury with reconstructions of representative means at $800 \mathrm{HU}$ show reduced $\mathrm{HO}$ formation in immobilized hind limb ( ${ }^{* *} P<0.001, n=3-4$ /group). (B) Confocal microscopy images of injured hind limb cross sections with indicated antibody probes at 1 week after injury with quantifications of FAK, pFAK, and TAZ (right) ( $n=3 /$ group, $n=1-3$ roi $/ \mathrm{mouse},{ }^{*} P<0.05$, ${ }^{*} P<0.01,{ }^{* * *} P<0.001$ ) (\#\#\# $P<0.0001$, Mann-Whitney $U$ test). Scale bars: $100 \mu \mathrm{m}$. Calculated using ANOVA (A) or Student's $t$ test (B).

differentiation, we sought to determine whether fiber alignment has the ability to skew MPC differentiation. MPCs were plated on aligned or nonaligned type 1 collagen-coated fibers in either nondifferentiating DMEM or a 1:1 mix of adipogenic and osteogenic media (termed mixed media). Cells were incubated for 72 hours, at which time they were harvested for RNA, and qPCR was performed. Cells plated on nonaligned fibers in mixed media had significantly increased expression of the early adipogenic marker Adipoq, whereas there was not a difference in the osteogenic marker Runx2 (Figure 6K). Aligned and nonaligned cell cultures in mixed media were performed for 7 days to assess the differences in lipid droplet accumulation, signifying mature adipocytes. Cells plated on nonaligned fibers had significantly greater lipid accumulation than those on the aligned fibers (Figure 6L). Collectively, 
A Uninjured

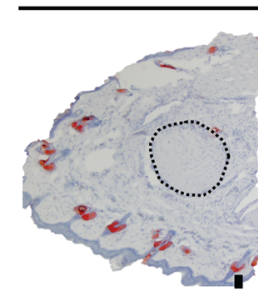

1 Week mobilized

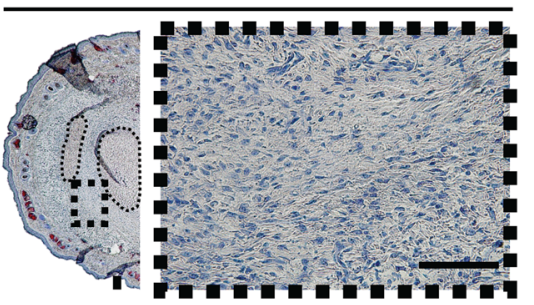

1 Week immobilized

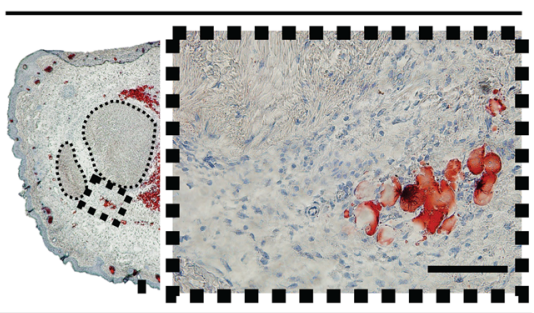

Oil Red O stain

B 1 Week mobilized

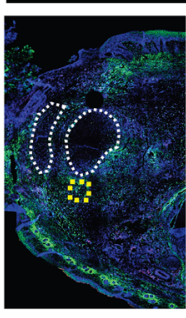

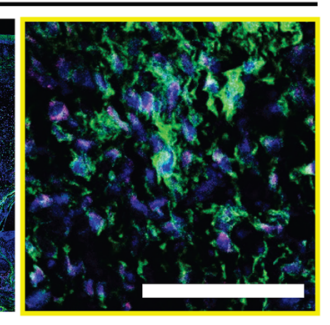

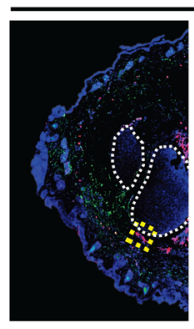

1 Week immobilized
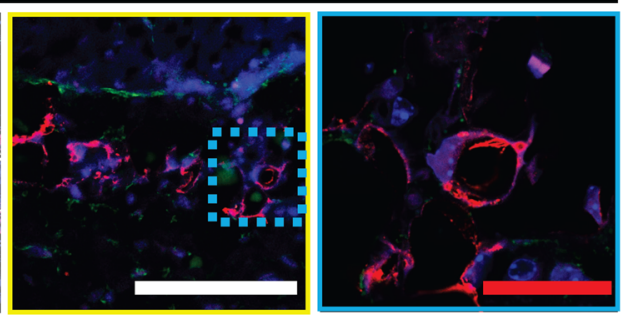

Hoechst PDGFRa Perilipin

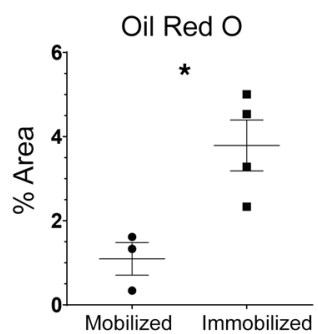

Perilipin

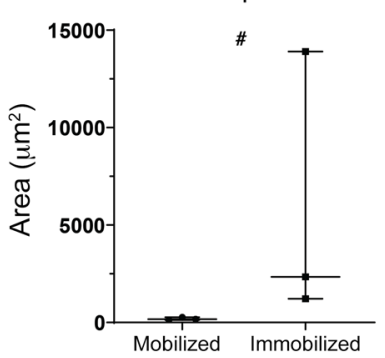

Figure 5. Immobilization increases adipogenesis at the $\mathrm{HO}$ anlagen. (A) Brightfield microscopy images of Oil Red $\mathrm{O}$ stained hind limb cross sections with quantification (right) ( $n=3-4 /$ group, $\left.{ }^{*} P<0.05\right)$. (B) Confocal microscopy images of injured hind limb cross sections with indicated antibody probes at 1 week after injury with quantification of perilipin ( $n=3$ /group, $\left.{ }^{\#} P<0.05\right)$. ${ }^{*}$ Calculated using Student's $t$ test, ${ }^{~}$ Calculated using Mann-Whitney $U$ test. Scale bars: $100 \mu$ m.

the data suggest that nonaligned ECM produced as a result of joint immobilization alters MPC spread and movement. Changes in cellular morphology affect TAZ signaling and skews MPC differentiation toward an adipogenic rather than osteogenic program. Interestingly, we found that FAK signaling was not affected by the changes in ECM fiber alignment, suggesting that changes in FAK during immobilization are occurring independent of alignment, adding to the complexity of how the microenvironment can modulate aberrant MPC fate after injury.

Immobilization results in genetic and epigenetic changes that alter MPC fate. To investigate genetic changes in MPCs after immobilization that might drive adipogenesis or osteogenesis, we performed both scRNA sequencing and single nuclei assay of transposase accessible chromatin (ATAC) sequencing on cells harvested from the extremity injury site of uninjured and mobile and immobilized mice 7 days after B/T. Canonical correlation analysis identified 14 unique clusters, all with characteristic profiles attributable to known cell types, with the exception of one small undefined cluster (Figure 7A). Cellular transcriptome data of the identified clusters were used to align open chromatin regions from the scATAC analysis. This allowed for the identification of the corresponding MPC population in the scATAC data (Figure 7B). To examine whether MPCs from immobile and mobile mice had different cell trajectories, analysis was performed on the cells from these 2 sample sets individually (Figure 7, C and D). Similar to the trajectory that included days 0,3 , 7, and 21 (Figure 1D), the trajectory from day 7 mobile mice resulted in terminal states of tenogenic, chondro/osteogenic, and adipogenic MPC cell fates (Figure 7C). However, MPCs from mice immobilized after B/T had terminal trajectory tenogenic and adipogenic states (Figure 7D). Next we analyzed the adipogenic and osteogenic potential of each of the cells. To do this, we compiled gene lists that were either associated with adipogenesis or osteogenesis signatures based on previous studies (Supplemental Table 1). These lists were then used to perform a correlation analysis where we could assign an adipogenic/osteogenic score to each cell. A score is calculated as the rank (Spearman) correlation between a vector of 78 adipogenic and 125 osteogenic genes (set to $1 /-1$, respectively), with the gene expression score calculated using the same gene set in each single cell. Correlation scores of each individual MPC from mobile or immobile mice are plotted in Figure 7E. MPCs coming from mice allowed to ambulate normally had a differentiation score more associated with osteogenesis, whereas MPCs coming from immobilized mice favored a more adipogenic signature (Figure 7E).

Examination of chromatin accessibility by scATAC sequencing revealed that immobile MPCs had decreased accessibility in Yap1 and Wwtrl (the gene encoding TAZ) (Figure 7F). Analyzing specific genes for differential accessibility from the list of genes used in our adipo/osteo correlation analysis revealed openness in adipogenic genes in MPCs from immobilized mice (Figure 7G) and osteogenic genes in mobile mice (Figure $7 \mathrm{H}$ ). Analysis of a set of genes associated with mechanotransduction revealed increased accessibility in Sox 2 and Cdc42 in immobile MPCs. Sox 2 is important in retaining cellular pluripotency (50) and $C d c 42$ has been implicated in mesenchymal cell senescence, and increased expression inhibits chondrogenic, osteogenic, and adipogenic differentiation (51). In addition to favoring adipogenesis, immobilization may also decrease MPC differentiation by retaining stemness or inducing cellular senescence. However, this will have to be investigated further. Additionally, other downstream targets of mechanotransduction important in adipogenic differentiation, namely Rapgef 1 and Rap1a, were more accessible in immobilized MPCs.

It was noted that there were differences in where mobile and immobile MPCs fell within the defined MPC cluster. We per- 
formed subclustering analysis and identified 5 subclusters of MPCs (Figure 8A). Based on the mobile and immobile composition of MPCs, we classified 3 groups from our subclusters: more mobile MPCs (clusters 0 and 3); mixed (both mobile and immobile) MPCs (clusters 1 and 2); and more immobile MPCs (cluster 4) (Figure 8A). Analysis of gene regions specifically open in the individual clusters of cells within the 3 groups was performed. Regions with a greater than 1.5-fold change were more closely examined. Genes around open regions associated with adipogenesis, osteogenesis, or stemness were highlighted. In group 2, those with a mix of mobile and immobile cells, open regions were seen in a mix of adipogenic, osteogenic, and stemness genes including Eif3h, Chrdl1, Lpl, and Pdgfra (Table 3). Both mobile and immobile MPCs were equally open in most of the identified genes (Figure 8B). In group 1, clusters containing more mobile than immobile MPCs, open regions were associated with genes important for osteogenesis, including Gas7, Runx2, and E2f3 (Table 3). Specifically, these osteogenic genes had more open chromatin in MPCs from the mobile mice (Figure 8B). Group 3, comprised mostly of MPCs from day 0 and immobilized mice, demonstrated open regions associated with genes for adipogenesis and stemness including Ncor2, Pbx1, and Sox6 (Table 3). Opposite to group 1, these gene regions were more open in MPCs coming from immobile mice (Figure 8B). Together, these data suggest that joint immobilization after severe injury results in decreased ECM alignment, leading to altered MPC mechanotransduction and changes in chromatin accessibility toward a signature that favors adipogenesis over osteogenesis, thus resulting in decreased formation of heterotopic ossification.

\section{Discussion}

In this study, we systematically demonstrate that mechanotransduction is important to the aberrant healing and MPC differentiation to bone that is seen in a mouse model of severe trauma (52). Further, we show that immobilization of the extremity after injury can alter ECM deposition and alignment, resulting in a dense, disorganized collagen network with increased stiffness at the location of extremity injury $(53,54)$. Further, we discovered that early immobilization after injury resulted in decreased $\mathrm{HO}$ formation and increased adipocyte deposition. In addition to changes in MPC fate, we noted changes in the cell shape/spread of MPCs present at the injury site, accompanied by decreased FAK and YAP/TAZ signaling. Inhibition of mechanotransductive FAK and YAP/TAZ signaling by genetic knockout resulted in decreased HO formation, further supporting the role of mechanotransduction in the formation of $\mathrm{HO}$ after severe injury. By either altering the ECM environment or blocking downstream mechanotransductive signaling, we were able to inhibit MPC osteogenic differentiation, suggesting that these 2 pathways could serve as attractive potential therapeutic options for the formation of extraskeletal bone after severe trauma injuries.

The ability of the MPCs to sense an extracellular mechanical stimulus as well as to translate this into an intracellular response through downstream signaling events is known as mechanotransduction (10). This signaling is accomplished through physical, biochemical, and biomechanical cues to the MPCs (9). Biochemical cues, such as the presentation of ligands and growth factors (GF) attached to the surface of ECM protein fibers like collagen and fibronectin, allow the cells to tether to the ECM through cell-surface receptors, called integrins, and provide growth signals to the MPCs $(11,55)$. Finally, physical and biomechanical cues, including mechanical properties of the ECM (stiffness, elasticity, and viscoelasticity), affect MPC behavior, including cell division, migration, and differentiation. ECM stiffness is a factor of both organization and composition $(11,56)$.

One specific signaling pathway is mediated by specific components of the focal adhesion complex (11). The FA is composed of machinery that determines how cells react to forces generated from the ECM (11). FAs link the ECM to the internal actin cytoskeleton via receptor-integrin interactions with the ECM outside and integrin-adaptor protein interactions inside the cell. FAK is then recruited to the FA and orchestrates downstream signaling events. Inhibiting FAK activation does not affect the formation of FAs; however, downstream Rac1-driven cellular processes such as cell migration are completely inhibited (20).

In addition to FAK signaling, other downstream signaling pathways of mechanotransduction have been proposed. Signaling through the transcriptional coactivators YAP1 and TAZ; YAP/TAZ has been shown to be an important mediator of cell differentiation in response to mechanical properties of the ECM such as stiffness and elasticity as well as changes in cell geometry and cytoskeletal organization (21-23). Stiffer in vitro substrates promote osteogenesis $(21,47,57,58)$ and nuclear translocation of YAP/TAZ, concurrent with increased transcriptional activity of YAP/TAZ regulated genes Ctgf and Ankrd1 (21). Osteogenic differentiation induced on stiff substrates is inhibited with depletion of Yap1/Wwtr1 by siRNA (21).

It has become increasingly recognized that the microenvironment created as a result of composition and stiffness of the ECM as well as growth factors sequestered in the surface of ECM protein fibers influence MPC fate and lineage commitment (11, $55,56,59)$. Previous studies have recognized FAK and YAP/TAZ signaling as important mediators controlling MPC differentiation (26, 60-63). YAP/TAZ activation has been recognized as the critical link between substrate stiffness and MPC differentiation into osteoblasts or adipocytes $(21,64,65)$. In vivo, FAK and YAP/ TAZ have been shown to be important in controlling bone marrow-derived MPC commitment to osteo- and chondrogenic fates (60-63). For example, in a model of mandibular distraction osteogenesis, FAK signaling played an important role in the formation of new bone (26). ECM stiffness has been extensively studied in vitro for its role in skewing MPC differentiation toward tissues that more closely resemble particular stiffnesses. For instance, differentiation into osteoblasts is favored by stiff environments that mimic natural bone (66), whereas softer matrices foster the development of an adipocyte cell fate $(57,67)$.

While these results seem contrary to those that we find in immobilized mice with regard to ECM stiffness, other factors that result from properties of the ECM can also impact MPC differentiation. It has been shown that cell shape and internal cytoskeletal structure significantly impact cellular function (68). McBeath et al. used microcontact printed ECM to demonstrate that cell spreading dictates the stem cell fate toward adipogenic or osteogenic programs (69). Inhibiting cell spreading has also been shown to result in decreased focal adhesion stability, decreased YAP/TAZ activity, and altered differentiation toward adipogene- 
A

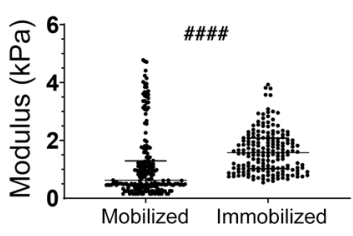

B

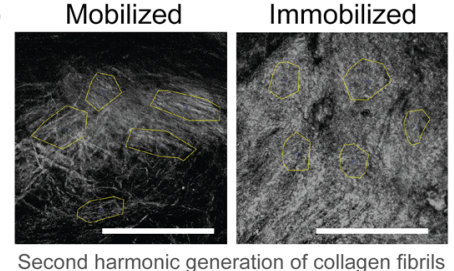

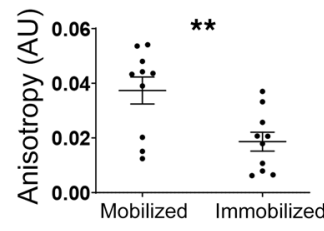

Mobilized Immobilized
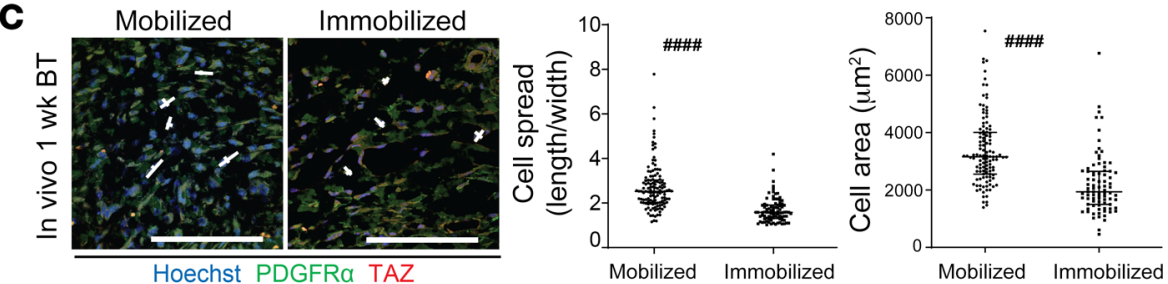

D
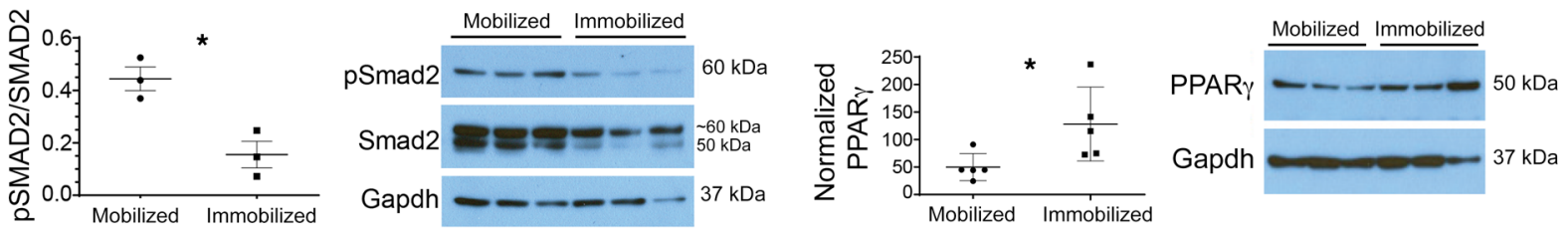

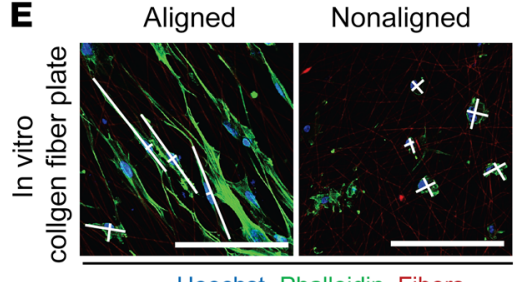

Hoechst Phalloidin Fibers

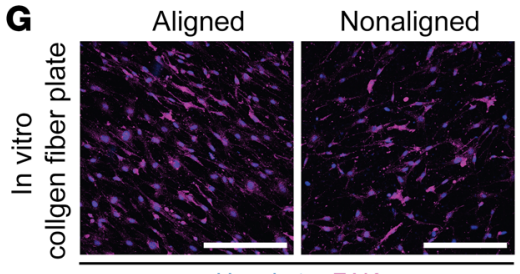

Hoechst pFAK

I

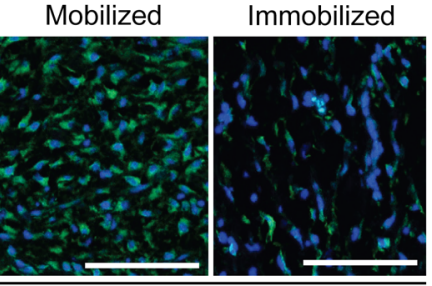

Hoechst PDGFRa
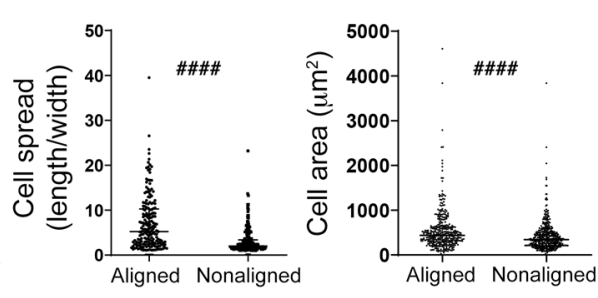

$\mathbf{F}$

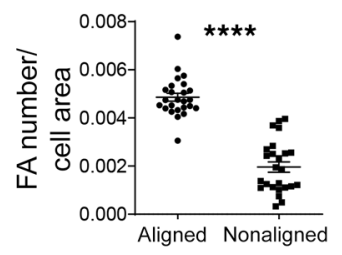

\section{H}
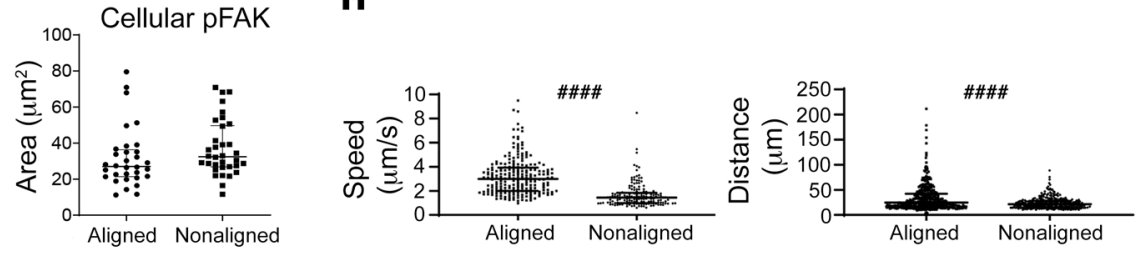

J
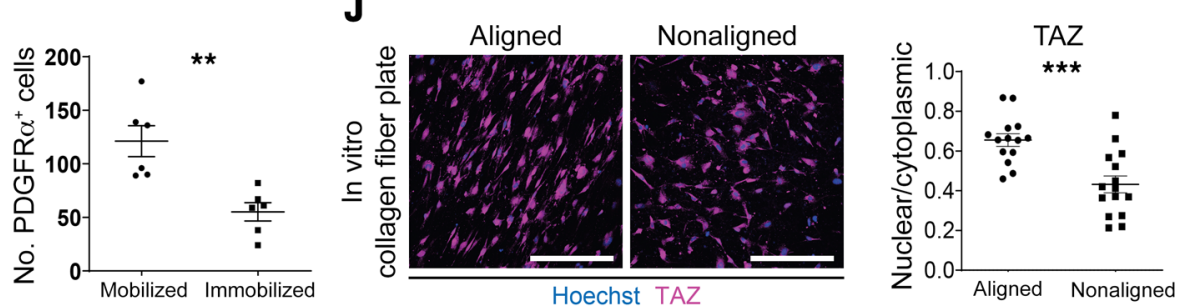

$\mathbf{K}$

Adipogenesis
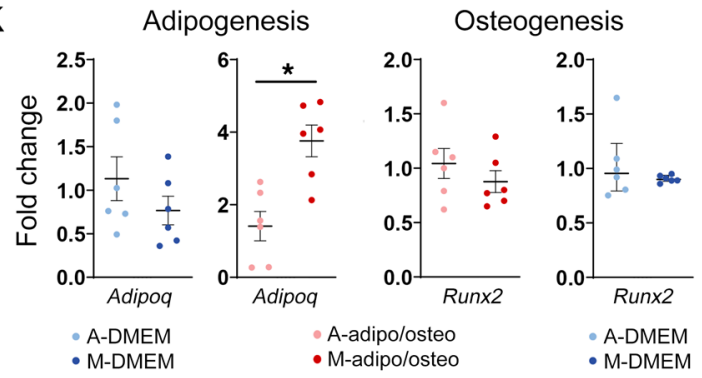

$\mathbf{L}$
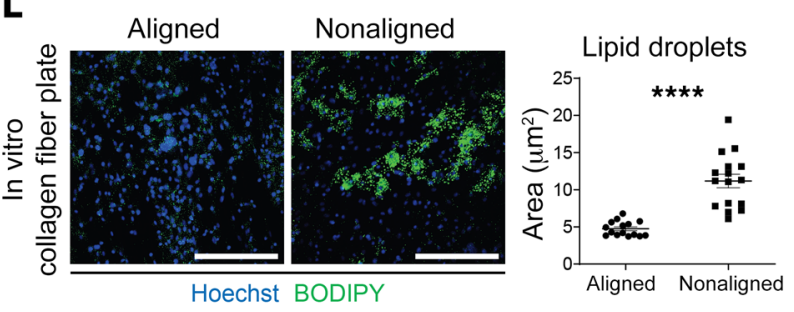
Figure 6. Immobilization alters the extracellular environment affecting MPCs. (A) Elastic modulus at site of $\mathrm{HO}$ formation immobilized and mobilized mice 1 week after $\mathrm{B} / \mathrm{T}$ as determined by atomic force microsco-

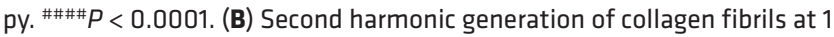
week after injury with anisotropy quantification (right) ( $n=2$ /group, ${ }^{* *} P$ $<0.01$ ). (C) Confocal microscopy images of hind limb cross sections 1 week after injury with indicated antibodies and quantified for cell spread and area (right) ( $n=3$ /group, \#\#\# $P<0.0001$ ). (D) Western blots for pSMAD2, SMAD2, PPAR $\gamma$, and GAPDH on whole-tissue lysate from 1-week post-B/T immobilized and mobilized mice. ${ }^{*} P<0.05$. (E) Confocal microscopy images of LST cells on aligned (A) and nonaligned (NA) collagen fiber plates probed with indicated antibodies and quantified for cell spread and area (right) ( $n=3 /$ group, $n=5$ roi/plate, \#\#\# $P<0.0001$ ). (F) Quantification of focal adhesions normalized by cell area of LST cells in aligned and nonaligned plates (**** $P<0.0001)$ (G) Confocal microscopy images of LST cells on aligned and nonaligned collagen fiber plates quantified for cellular pFAK (10-12 cells/n, $n=3 /$ group). (H) Quantification of speed and distance traveled by LSTs on aligned and nonaligned plates: Supplemental Video 1 (\#\#\#P< 0.0001$)$. (I) Confocal microscopy images of hind limb cross sections at 1 week after injury quantified for number for number of PDGFR ${ }^{+}$ cells. ${ }^{*} P<0.01$. (J) Confocal microscopy images of LST cells on aligned and nonaligned collagen fiber plates probed for TAZ and quantified for nuclear/ cytoplasmic ratio (right) (4-5 images/n, $n=3 /$ group, ${ }^{* * *} P=0.0003$ ). (K) Effects of aligned (A) or nonaligned $(\mathrm{N})$ electrospun collagen I coated fibers on Runx2 and Adipoq expression in either DMEM or mixed medium ( $n=$ 3 /group, ${ }^{*} P<0.05$, A vs. N within media type). (L) Confocal microscopy images of LST cells on aligned and nonaligned collagen fiber plates in mixed medium for 7 days and subsequently stained with BODIPY and lipid droplets quantified (right) (5-6 images/n, $n=3$ /group, ${ }^{* * *} P<0.0001$ ). *Calculated using Student's $t$ test, "Calculated using Mann-Whitney $U$ test. Scale bars: $100 \mu \mathrm{m}$.

sis $(21,49)$. It has also been shown that inhibition of cell spreading antagonized BMP2-induced osteogenesis (70). Further, David et al. demonstrated that heightened cell contractility, a mediator between mechanical cues and YAP/TAZ signaling (71), inhibited adipogenic commitment and promoted osteogenesis through the regulation of transcription factor Ppary (72). Although these studies were limited, using only 2D cell culture systems lacking ECM, Tang et al. demonstrated in a 3D system as well as in vivo the importance of progenitor cell shape, remodeling of ECM, and $\mathrm{YAP} / \mathrm{TAZ}$ signaling in redirecting skeletal stem cell differentiation from osteogenesis toward adipogenesis (62). Our immobilization results support these previous findings, where we see decreased cell spreading, supporting adipogenesis over osteogenesis.

In this study we used an in vivo mouse model of severe trauma to demonstrate that joint immobilization, a noninvasive and nonpharmacological modality, results in altered physical and biomechanical properties of the ECM and changes MPC fate. Additionally, in this context, in vivo immobilization allowed us to study the role ECM alignment has in regard to cell migration and differentiation in the context of wound healing. This intervention is important in situations where MPC differentiation in the context of tissue repair goes awry (eg, $\mathrm{HO}$ and muscle fibrosis). Whereas previous reports have suggested that a stiffer matrix stimulates MPC osteogenic differentiation $(21,57,58)$, our data suggest that ECM alignment and cell spreading, and not stiffness per se, dictates MPC fate after extremity injury. The data presented here are an important step in the field of treating extremity injury and help to explain the molecular basis of this modality that has previously been undescribed. In the context of severe trauma resulting in aberrant wound healing leading to ectopic bone formation, the current study highlights the potential use of immobilization and pharmacological inhibitors to block downstream mechanotransductive pathways. Early stages of HO induce mechanotransductive pathways that activate downstream osteogenic pathways in progenitor cells that ultimately lead to ectopic bone formation $(73$, 74). The current studies indicate that in injured tissues, immobilization through its ability to alter mechanotransduction leads to benign adipogenesis rather than debilitating osteogenesis in MPCs, which has substantial clinical implications. Furthermore, the use of pharmacologic inhibitors that alter mechanotransductive signaling, like PF573228, which blocks FAK signaling, may prove to be effective therapies that similarly induce adipogenesis at sites prone to osteogenesis. It should be noted that the accumulation of adipose tissue in the joints near the injury site is far less debilitating than HO, resulting in far more favorable outcomes (75). Furthermore, the overgrowth of adipose within soft tissue mimics intramuscular lipomas, which have been shown to have good response to surgical debulking without recurrence or the need for more invasive therapies in the future (75). Together, these findings demonstrate the potential clinical application of immobilization and the use of therapies that alter mechanotransduction, including FAK inhibitors, for clinical treatment of trauma-induced aberrant ectopic bone formation.

\section{Methods}

Animal use. All animals were housed in IACUC-supervised facilities at $18^{\circ} \mathrm{C}-22^{\circ} \mathrm{C}$ on a 12 -hour light-dark cycle with ad libitum access to food and water. For all in vitro and in vivo studies requiring WT mice, young adult male (6-10 weeks old) C57BL/6J mice were purchased from Jackson Laboratory. PrxCre FAK ${ }^{\mathrm{f} / \mathrm{l} l}$, Hoxa11CreER Yap ${ }^{\mathrm{f} / \mathrm{fl}} \mathrm{Taz}^{\mathrm{f} / \mathrm{l} \mathrm{l}}$, and Hoxa11CreER R26 $6^{\text {TdTomato }}$ mice were bred in house. PrxCre mice obtained from the laboratory of Ernestina Schipani (University of Michigan, Ann Arbor, Michigan, USA) were crossed with Ptk2(FAK) ${ }^{\mathrm{fl} / \mathrm{l}}$ mice that were obtained from Teitelbaum's lab (University of Michigan, Ann Arbor, Michigan, USA). Hoxa11CreER mice were obtained from Deneen Wellik (University of Wisconsin, Madison, Wisconsin, USA). $Y a p^{\mathrm{f} / \mathrm{fl}} \mathrm{Taz}^{\mathrm{fl} / \mathrm{l}}$ mice were a gift from Stephen J. Weiss (Life Sciences Institute, University of Michigan, Ann Arbor, Michigan, USA). Hoxa11CreER R26 $6^{\text {TdTomato }}$ and the Hoxa11CreER Yap ${ }^{\mathrm{f} / \mathrm{fl}} \mathrm{Ta}^{\mathrm{f} / \mathrm{fl}}$ mouse lines were then bred in-house in our mouse facility at the University of Michigan.

Burn/tenotomy HO model. A partial-thickness scald burn injury was administered to animals according to a previously described protocol by a single surgeon (refs. 53, 54 and Supplemental Methods).

Joint immobilization. Joint immobilization was performed immediately after injury as previously described (39). Briefly, the immobilizer was prepared from a $1.5 \mathrm{~mL}$ Eppendorf tube. The lid was removed and the edge was smoothed using a razor blade. The end of the tube was removed to allow for air flow around the limb. A piece of sponge was applied to the proximal end using Coband to allow for padding to prevent pressure ulceration against the body. Legs were placed into the immobilizers with the foot in plantar-flexion and the knee joint in extension, and the immobilizers were anchored to the body using superglue (Supplemental Figure 3A).

Human HO immunohistochemistry. Forty-five cases of nonhereditary HO were identified in our surgical pathology archives (Johns Hopkins University, Baltimore, Maryland, USA). Cases were obtained 
A

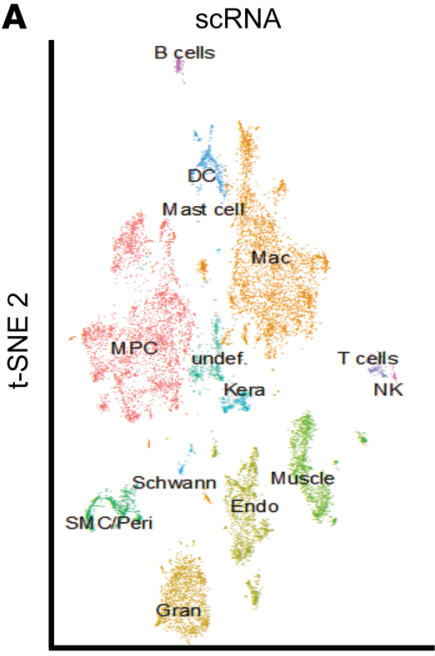

t-SNE 1

D

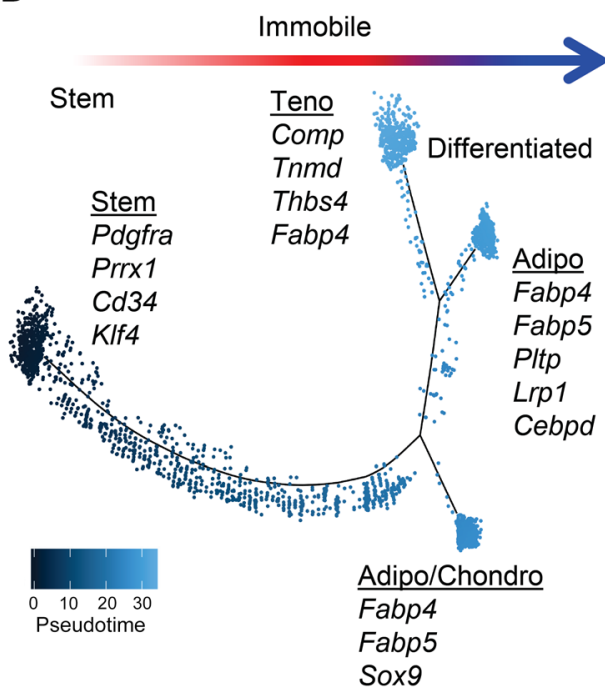

B

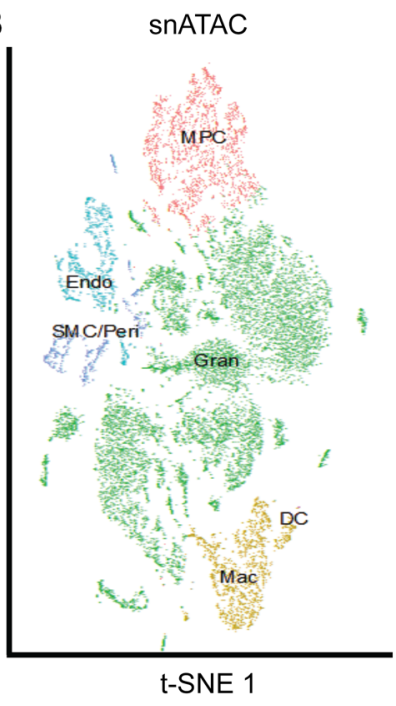

E
C

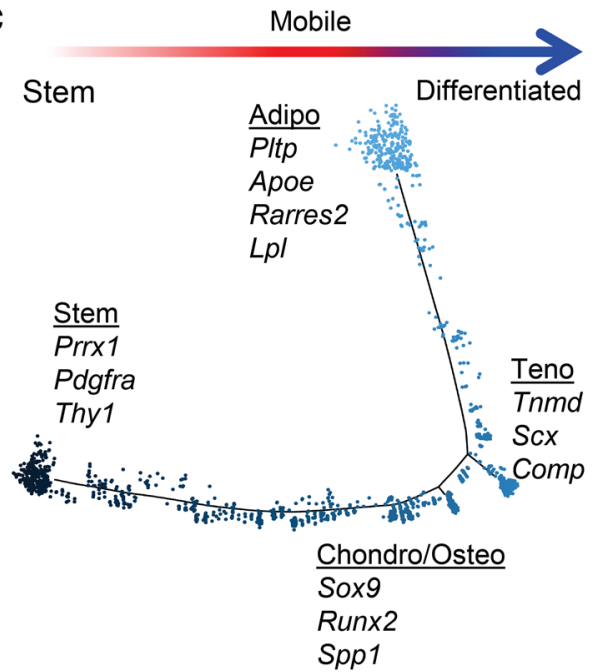

Osteogenic
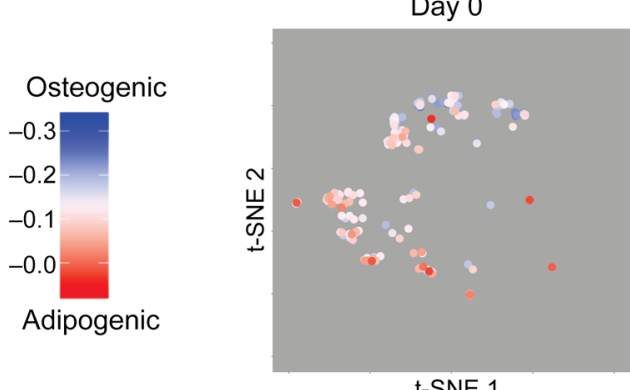

t-SNE 1

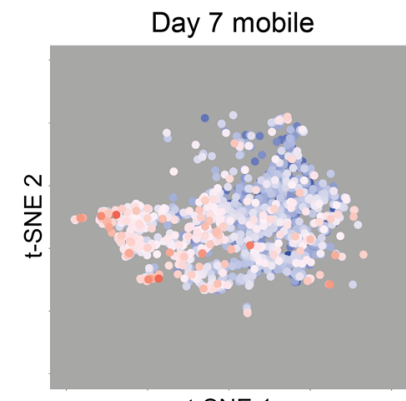

t-SNE 1

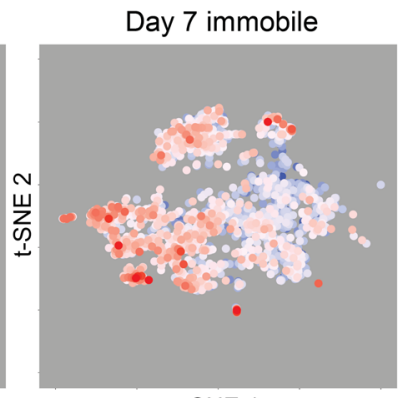

t-SNE 1

$\mathbf{F}$

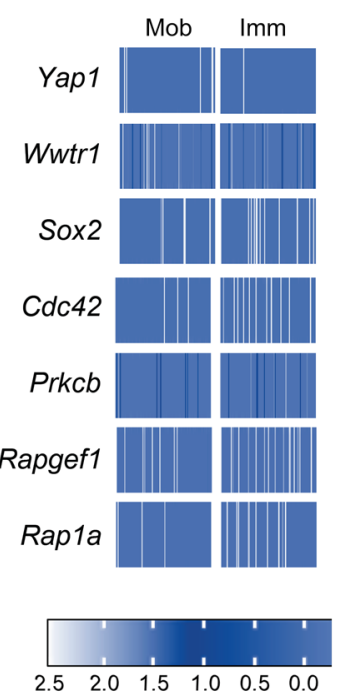

G

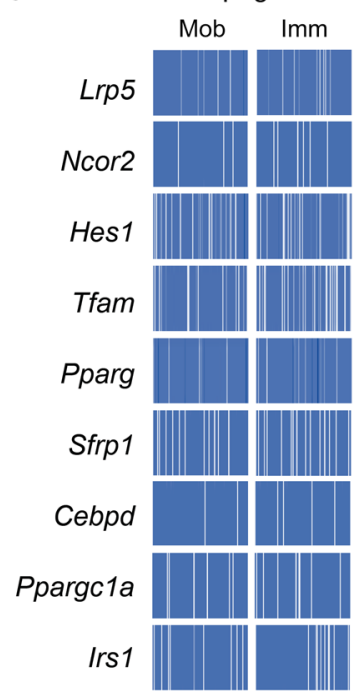

H

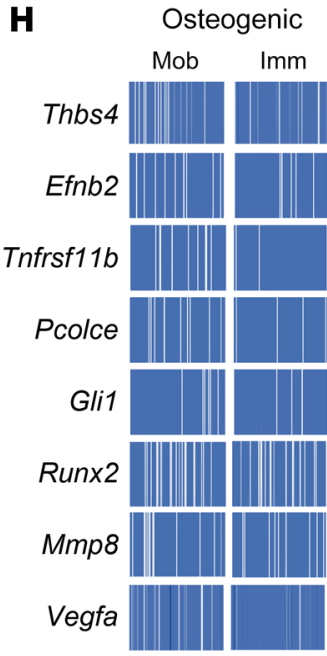

Figure 7. Immobilization results in genetic changes that alter MPC fate. (A) Canonical correlation analysis of cells from the $\mathrm{HO}$ site of day 7 postinjury mobile and immobile mice defines 14 clusters, including an MPC subset. (B) MPCs from sCATAC sequencing were identified based on RNA expression in the scRNA-seq results. Trajectory analysis of MPCs from (C) mobile and (D) immobile mice across pseudotime. (E) Adipogenic/osteogenic expression scores calculated on a per MPC basis from the clusters identified in the scRNA-seq analysis of day 0 naive, day 7 mobile, and day 7 immobilized mice. Chromatin accessibility in gene regions specific to the MPC cluster represented by heatmaps of the average log fold change differences in (F) mechanotransduction genes, (G) adipogenic genes, or $(\mathbf{H})$ osteogenic genes compared with locations in other clusters. Heatmaps display the openness in 100 sampled cells from either mobile (Mob) or immobile (Imm) mice that contribute to the MPC cluster from the sCATAC-seq analysis. 
A

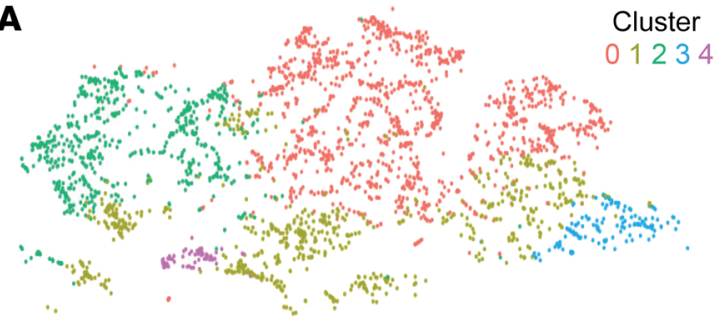

B

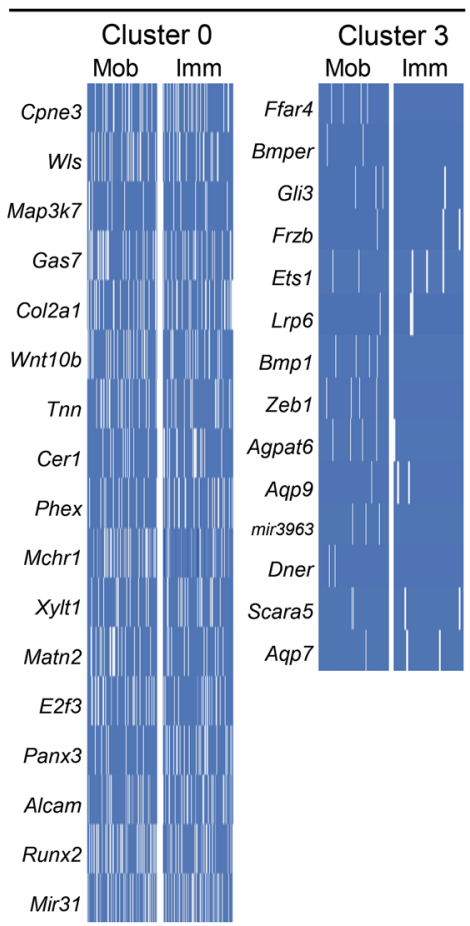

Group 2
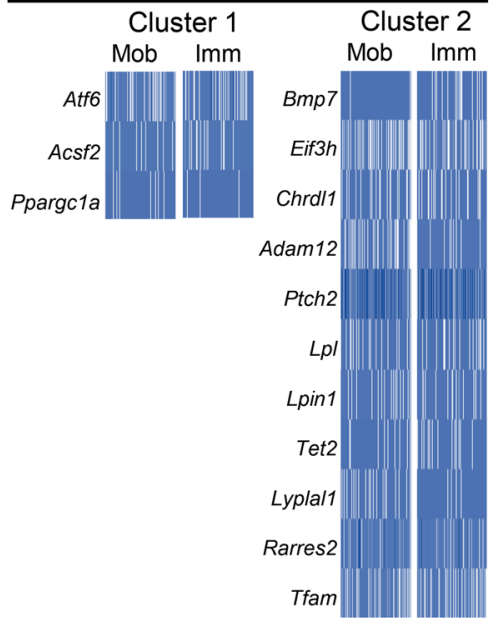

Group 3

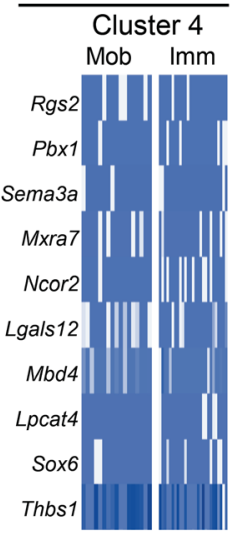

Figure 8. scATAC-seq MPC subclusters reveal distinct MPCs from mobile and immobile mice. (A) Subcluster analysis of the MPCs from the SCATAC-seq results identifies 5 subclusters: group 1 comprises clusters 0 and 3 ; group 2 comprises clusters 1 and 2; and group 3 comprises cluster 4 . On day 0 , the number of MPCs in clusters $0-4$ was $11,91,103,1$, and 30 , respectively. In day 7 mobile mice, the number of MPCs in clusters $0-4$ was 416 $290,240,40$, and 27 , respectively. In day $7 \mathrm{immobile}$ mice, the number of MPCs in clusters $0-4$ was $780,292,260,106$, and 17, respectively. (B) Heatmaps representing marker genes for each cluster in groups 1, 2, and 3. Group 1: more Mob MPCs; group 2: mixed Mob and Imm; group 3: more $1 \mathrm{~mm}$ and day 0 . Heatmap scale is average log fold change difference in chromatin accessibility between listed cluster compared with other MPC clusters from 100 sampled cells (or number of cells available) from either mobile or immobile mice contributing to each of the MPC subclusters. under IRB approval with waiver of informed consent. All material was coded so as to protect the confidentiality of personal health information. Of the cases for which tissue blocks were available, 2 of 45 showed early, granulation tissue-like stages of HO. For each, $10-\mu \mathrm{m}$-thick sections were prepared and pretreated with xylene and different concentrations of ethanol. Antigen retrieval was performed by using trypsin enzymatic antigen retrieval solution (catalog ab970, Abcam) for 15 minutes at room temperature. After washing in PBS, the sections were incubated in 3\% hydrogen peroxide for 20 minutes for blocking with endogenous peroxidase and followed by washing with PBS. Next, the slides were blocked with $5 \%$ goat serum (catalog S-1000, Vector Laboratories) for 30 minutes and then probed with $0.1 \%$ PBST-diluted rabbit anti-phosphoFAK (pFAK) (catalog ab40794, Abcam) in 1:250 concentration overnight at $4^{\circ} \mathrm{C}$. The next day, the primary antibody was washed off with $0.01 \%$ PBST and the slides were probed with $0.01 \%$ PBST diluted anti-rabbit IgG in 1:200 concentration for 1 hour. After applying both VECTASTAIN ABC kit and DAB Peroxidase Substrate (catalog SK-4100, Vector Laboratories) the samples were visualized. The slides were then rinsed thoroughly in PBS before reblocking with BIOXALL Endogenous Peroxidase and Alkaline Phosphatase Blocking Solution (catalog SP-6000, Vector Laboratories) for 10 minutes and then $2.5 \%$ normal house serum for 20 minutes at room temperature. After reblocking the slides, 0.1\% PBST diluted mouse anti-
PDGFRA primary antibodies in 1:200 concentration was applied (Abcam, catalog ab96569). After PBS wash, ImmPRESS AP AntiMouse IgG (catalog MP-5402, Vector Laboratories) was applied for 30 minutes at room temperature and then VECTOR Red Alkaline Phosphatase Substrate Kit (catalog SK-5100, Vector Laboratories) was applied for red color visualization. Hematoxylin counterstaining was performed at the end for nuclear staining.

Second harmonic generation imaging. Multiphoton (MP) microscopic imaging was used to survey collagen at the injury site, due to its highly crystalline, noncentrosymmetric structure, as previously described (76). Cryopreserved $10 \mu \mathrm{m}$ sections on slides were washed in $0.05 \%$ TBST to remove OCT from section. Slides were then mounted with water-based mounting media, covered with glass coverslips, and sealed with nail polish. Slides were imaged using a Leica SPX8 confocal laser scanning microscope. The pinhole was opened to maximum diameter. The multiphoton laser was set to $860 \mathrm{~nm}, 66 \%$ offset, $60 \%$ gain, and 20\% intensity. HyD1 detector was set to $420-440 \mathrm{~nm}$. Images were taken of pre-HO anlagen adjacent to injured tendon. For downstream analysis of fibrillar structures, we used a previously validated ImageJ plugin, FibrilTool, to quantify array orientation and anisotropy as determined from eigenvectors of the Hessian matrix (77). Multiple regions of interest for analysis were selected, taking care to avoid regions of saturation as prescribed by the software authors $(n=5 /$ spec- 
Table 3. Genes in the scATAC-seq MPC subclusters

\begin{tabular}{|c|c|c|c|c|c|c|c|c|c|}
\hline $\begin{array}{l}\text { Genes, } \\
\text { subcluster } 0 \text {, } \\
\text { group } 1\end{array}$ & $\begin{array}{l}\text { Average } \\
\log \mathrm{FC}\end{array}$ & $\begin{array}{c}\text { Genes, } \\
\text { subcluster 1, } \\
\text { group } 2\end{array}$ & $\begin{array}{c}\text { Average } \\
\log \mathrm{FC}\end{array}$ & $\begin{array}{c}\text { Genes, } \\
\text { subcluster } 2 \text {, } \\
\text { group } 2\end{array}$ & $\begin{array}{c}\text { Average } \\
\log \mathrm{FC}\end{array}$ & $\begin{array}{c}\text { Genes, } \\
\text { subcluster } 3 \text {, } \\
\text { group } 1\end{array}$ & $\begin{array}{l}\text { Average } \\
\text { log FC }\end{array}$ & $\begin{array}{l}\text { Cenes, } \\
\text { subcluster } 4 \text {, } \\
\text { group3 }\end{array}$ & $\begin{array}{l}\text { Average } \\
\log \mathrm{FC}\end{array}$ \\
\hline [pne $^{\mathrm{A}}$ & 0.89154286 & Atf $\sigma^{A}$ & 0.54365239 & $B m p 7^{A}$ & 0.67905232 & Clij $^{\mathrm{A}}$ & 1.28562529 & Sema3aA & 1.19931204 \\
\hline $\operatorname{Map} 3 k 7^{A}$ & 0.76478184 & Ppargc1a & 0.52600873 & Chrd/1/ & 0.65763083 & Ets $1^{A}$ & 0.87914107 & Bmpr1b $^{A}$ & 0.44504581 \\
\hline $\operatorname{Cas} 7^{A}$ & 0.41722132 & Chd $4^{\mathrm{C}}$ & 0.4334688 & 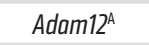 & 0.44823405 & $L r p 6^{A}$ & 0.82502347 & Ppargc1a & 1.22941412 \\
\hline $\mathrm{Col} 2 a 1^{\mathrm{A}}$ & 0.41254696 & Pdgfra ${ }^{C}$ & 0.5217095 & $\operatorname{Ptch}^{\mathrm{A}}$ & 0.41455012 & $B m p 1^{A}$ & 0.75667322 & Ncor2 ${ }^{\mathrm{B}}$ & 1.14134454 \\
\hline$T n n^{\AA}$ & 0.69880899 & & & $\operatorname{Lpin} 1^{B}$ & 0.7946399 & Agpat $^{B}$ & 0.78560715 & $M b d 4^{B}$ & 0.60261142 \\
\hline Cer $]^{A}$ & 0.6935433 & & & Tet $2^{\mathrm{B}}$ & 0.74108949 & AqpgB & 0.77823255 & Lpcat4 $4^{\mathrm{B}}$ & 0.58580296 \\
\hline Phex ${ }^{A}$ & 0.68083317 & & & Lyplal1 & 0.62471079 & Mir3963ㄹ & 0.75060097 & Sox $6^{\mathrm{B}}$ & 0.55829541 \\
\hline$M c h r 1^{A}$ & 0.64614796 & & & Rarres2 $^{\mathrm{B}}$ & 0.49878574 & $D n e r^{B}$ & 0.68236705 & Thbs $1^{\mathrm{B}}$ & 0.40406076 \\
\hline$X y \mid t t^{A}$ & 0.6454567 & & & Tfam $^{B}$ & 0.48435695 & Scara $5^{B}$ & 0.53780159 & $\operatorname{Rgs} 2^{\mathrm{B}}$ & 0.8944518 \\
\hline $\operatorname{Matn} 2^{A}$ & 0.64396668 & & & Pdgfra ${ }^{\mathrm{A}}$ & 0.47067238 & $A q p 7^{B}$ & 0.48094055 & $P b x 1^{B}$ & 0.50557775 \\
\hline Runx $2^{A}$ & 0.54856932 & & & & & $\operatorname{Tm} 4 s f 1^{\mathrm{C}}$ & 0.91012344 & $T n m d^{0}$ & 1.35910891 \\
\hline Mir31 & 0.57320291 & & & & & Сура & 0.9001523 & Eya $2^{0}$ & 1.18132022 \\
\hline Parp ${ }^{\complement}$ & 0.54709244 & & & & & $N r 2 f 2^{C}$ & 0.79881738 & $F \bmod 1^{D}$ & 0.60183199 \\
\hline
\end{tabular}

${ }^{A}$ Osteogenic. ${ }^{B}$ Adipogenic. ${ }^{\text {CStemness. }}{ }^{\text {DTenocyte. }}$

imen, 3 biological specimens/group, sampled regions $N=15 /$ group) to produce anisotropy values and mean orientation.

Atomic force microscopy. Legs were harvested and watercolor marked. Samples were flash frozen and placed at $-20^{\circ} \mathrm{C}$ overnight, region of interest was excised with a razor blade and promptly embedded. Samples were chilled at $-80^{\circ} \mathrm{C}$ for at least 1 hour before sectioning. Leica Cryostat was used to section samples at $20 \mu \mathrm{m}$ thickness onto $0.01 \%$ poly-L-lysine glass coverslips, which were stored in a $-80^{\circ} \mathrm{C}$ freezer. To prepare the samples for microscopy, OCT was washed off using 1× PBS (Gibco). A permanent marker was used on the slide to mark gross regions of interest. Samples were adhered to glass slides with clear nail polish and cured for 10 minutes before probing samples. Regions of interest were determined using slide markings, anatomy, and watercolor tissue marking. A cantilever (HYDRA6V-200NG-10, AppNano) with an attached glass microsphere (15 $\mu \mathrm{m}$ radius) was calibrated before obtaining force curves on tissue in $1 \times$ PBS. Four regions, 16 force curves/region, were obtained for each sample. Hertz spherical model for indentation was used to find the elastic modulus of tissue. Force curves were analyzed using AtomicJ, assuming a Poisson's ratio of 0.5. Noncurves were not included.

Fabrication of fibrous matrices. Dextran (MW 86,000 Da, MP Biomedicals) was methacrylated by reaction with glycidyl methacrylate as previously described (78). Briefly, $10 \mathrm{~g}$ dextran and $1 \mathrm{~g} 4$-dimethylaminopyridine was dissolved in $50 \mathrm{~mL}$ dimethylsulfoxide (DMSO) under vigorous stirring for 8 hours. Glycidyl methacrylate $(25.0 \mathrm{~mL})$ was then added and the reaction mixture was heated to $45^{\circ} \mathrm{C}$ for 24 hours. The solution was cooled at $4^{\circ} \mathrm{C}$ for 1 hour and precipitated into $1 \mathrm{~L}$ ice-cold 2-isopropanol. The crude product was recovered by centrifugation, redissolved in milli-Q water, and dialyzed against milli-Q water for 3 days. To create heparin methacrylate (HepMA), heparin sodium salt was reacted with methacrylic anhydride following pre- viously described procedures (79). Briefly, heparin sodium salt (500 $\mathrm{mg}$ ) was dissolved in $50 \mathrm{~mL}$ PBS under vigorous stirring before addition of methacrylic anhydride $(99.3 \mathrm{~mL})$. The reaction was kept under constant stirring at $4^{\circ} \mathrm{C}$ for 24 hours. $\mathrm{NaOH}(1 \mathrm{~N})$ was added every hour for the first 6 hours to maintain a solution $\mathrm{pH}$ of 8 . The product was dialyzed against milli-Q water for 3 days and then lyophilized. Final products were characterized by H-NMR.

Electrospun dextran methacrylate (DexMA) fibers were generated as previously described (80). In brief, DexMA was dissolved at $0.50 \mathrm{~g} / \mathrm{mL}$ in a 1:1 mixture of milli-Q water and dimethylformamide with $0.005 \%$ Irgacure 2959 photoinitiator. This polymer solution was used for electrospinning within an environment-controlled glovebox held at $21^{\circ} \mathrm{C}$ and $30 \%$ relative humidity. Electrospinning was performed at a flow rate of $0.5 \mathrm{~mL} / \mathrm{h}$, a gap distance of $8 \mathrm{~cm}$, and a voltage of $-5.0 \mathrm{kV}$, onto a collecting surface of oppositely charged $(5.0 \mathrm{kV})$ parallel electrodes with varying separation distance to control alignment. Methacrylated rhodamine was incorporated into the electrospinning solution to fluorescently visualize DexMA fibers. Fiber samples were first primary cross-linked under UV light $\left(100 \mathrm{~mW} / \mathrm{cm}^{2}\right)$ and then hydrated in a photoinitiator solution of 1 $\mathrm{mg} / \mathrm{mL}$ Irgacure 2959 in milli-Q water and exposed to a secondary cross-linking of UV light $\left(100 \mathrm{~mW} / \mathrm{cm}^{2}\right)$ for 30 seconds to stabilize networks. To enable cell adhesion, heparin methacrylate was dissolved secondary solutions at $2.5 \%$ (wt/vol) followed by a 30 minute incubation in $50 \mu \mathrm{g} / \mathrm{mL}$ type- 1 collagen. For all studies, cells were trypsinized, counted, and seeded onto substrates at a density of 1500 cells $/ \mathrm{cm}^{2}$, unless specified otherwise.

Data and materials availability. scRNA sequencing data publicly available on GEO (GSE126060). scRNA and scATAC sequencing data from the mobile and immobile mice are accessible on the Gene Expression Omnibus database (accession no. GSE150995). 
Statistics. More information about statistics is available in the Supplemental Methods.

Study approval. All animal procedures were carried out in accordance with the guidelines provided in the Guide for the Use and Care of Laboratory Animals (National Academies Press, 2011) and were approved by the IACUC of the University of Michigan (PRO0007930).

\section{Author contributions}

AKH planned and executed experiments, analyzed results, and wrote the manuscript. NP executed experiments, composed figures, and critically reviewed the manuscript. CP: executed experiments, composed figures, and critically reviewed the manuscript. SM performed scRNA sequencing analysis and critically reviewed the manuscript. DLM and MS executed experiments and critically reviewed the manuscript. $\mathrm{CH}$ performed immunofluorescence and SHG imaging. CYH performed human IHC. AP performed DMA analysis and critically reviewed the manuscript. NDV provided assistance with experiments. ALS wrote and critically reviewed/edited the manuscript. JAG and RN performed microCT splining and analysis. SL performed cell culture experiments. MTL and SJW gave input on experiments and critically reviewed the manuscript. YT generated and characterized the YAP/TAZ knockout mice. BMB gave input on experiments and critically reviewed/ edited the manuscript. AWJ obtained human IHC samples, gave input on the experiments, and critically reviewed/edited the manuscript. BL oversaw the project and along with AKH planned experiments, analyzed data, wrote, and critically reviewed and edited the manuscript.

\section{Acknowledgments}

We would like to extend our thanks to Ellen Arruda for use of equipment and assistance. Work described in this manuscript was performed using our biomedical core facilities. We would like to acknowledge the Advanced Genomics Core, Epigenomics Core, and the Flow Cytometry Core at the University of Michigan, Ann Arbor, Michigan, USA. This work was supported by funding from $\mathrm{NIH} /$ National Institute of Arthritis and Musculoskeletal and Skin Diseases (NIAMS) NIH1R01AR071379, NIH/National Institute of General Medical Sciences NIHR01GM123069, and the American College of Surgeons Clowes Award (to BL). Research reported in this publication was supported by NIAMS Award P30 AR069620 (to AP) and an International Fibrodysplasia Ossificans Progressiva Association (IFOPA) Accelerating Cures and Treatments (ACT) grant (to AKH). The content is solely the responsibility of the authors and does not necessarily represent the official views of the NIH.

Address correspondence to: Benjamin Levi and Amanda Huber: 1150 West Medical Center Drive, MSRBII Room A574D, Ann Arbor, Michigan 48109, USA. Phone: 734.936.5895; Email: blevi@ med.umich.edu (BL). Phone: 734.763.1949; Email: hubera@ umich.edu (AH).
1. Ootes D, Lambers KT, Ring DC. The epidemiology of upper extremity injuries presenting to the emergency department in the United States. Hand (N Y). 2012;7(1):18-22.

2. Lambers K, Ootes D, Ring D. Incidence of patients with lower extremity injuries presenting to US emergency departments by anatomic region, disease category, and age. Clin Orthop Relat Res. 2012;470(1):284-290.

3. Banerjee M, et al. Epidemiology of extremity injuries in multiple trauma patients. Injury. 2013;44(8):1015-1021.

4. Kunz RI, Coradini JG, Silva LI, Bertolini GR, Brancalhão RM, Ribeiro LF. Effects of immobilization and remobilization on the ankle joint in Wistar rats. Braz JMed Biol Res. 2014;47(10):842-849.

5. Lamb SE, Marsh JL, Hutton JL, Nakash R, Cooke MW, Collaborative Ankle Support Trial (CAST Group). Mechanical supports for acute, severe ankle sprain: a pragmatic, multicentre, randomised controlled trial. Lancet. 2009;373(9663):575-581.

6. Nakagawa Y, et al. Duration of postoperative immobilization affects MMP activity at the healing graft-bone interface: Evaluation in a mouse ACL reconstruction model. JOrthop Res. 2019;37(2):325-334.

7. Gallagher BP, Bishop ME, Tjoumakaris FP, Freedman KB. Early versus delayed rehabilitation following arthroscopic rotator cuff repair: A systematic review. Phys Sportsmed. 2015;43(2):178-187.

8. Jackson M, Tétreault P, Allard P, Begon M. Optimal shoulder immobilization postures following surgical repair of rotator cuff tears: a simulation analysis. J Shoulder Elbow Surg. 2013;22(8):1011-1018.
9. Gattazzo F, Urciuolo A, Bonaldo P. Extracellular matrix: a dynamic microenvironment for stem cell niche. Biochim Biophys Acta. 2014;1840(8):2506-2519.

10. Jansen KA, Atherton P, Ballestrem C. Mechanotransduction at the cell-matrix interface. Semin Cell Dev Biol. 2017;71:75-83.

11. DuFort CC, Paszek MJ, Weaver VM. Balancing forces: architectural control of mechanotransduction. Nat Rev Mol Cell Biol. 2011;12(5):308-319.

12. Agarwal S, et al. Inhibition of Hif1 $\alpha$ prevents both trauma-induced and genetic heterotopic ossification. Proc Natl Acad Sci USA. 2016;113(3):E338-E347.

13. Kan C, et al. Gli1-labeled adult mesenchyma stem/progenitor cells and hedgehog signaling contribute to endochondral heterotopic ossification. Bone. 2018;109:71-79.

14. Kan L, Peng CY, McGuire TL, Kessler JA. Glastexpressing progenitor cells contribute to heterotopic ossification. Bone. 2013;53(1):194-203.

15. Dey D, et al. Two tissue-resident progenitor lineag es drive distinct phenotypes of heterotopic ossification. Sci Transl Med. 2016;8(366):366ra163.

16. Lees-Shepard JB, et al. Activin-dependent signaling in fibro/adipogenic progenitors causes fibrodysplasia ossificans progressiva. Nat Commun . 2018;9(1):471.

17.Agarwal S, et al. BMP signaling mediated by constitutively active Activin type 1 receptor (ACVR1) results in ectopic bone formation localized to distal extremity joints. Dev Biol. 2015;400(2):202-209.

18.Agarwal S, et al. Scleraxis-lineage cells contribute to ectopic bone formation in muscle tendon. Stem Cells. 2016;35(3):705-710.
19. Levi B, et al. Risk factors for the development of heterotopic ossification in seriously burned adults: A National Institute on Disability, Independent Living and Rehabilitation Research burn model system database analysis. J Trauma Acute Care Surg. 2015;79(5):870-876.

20. Horton ER, et al. Modulation of FAK and Src adhesion signaling occurs independently of adhesion complex composition. J Cell Biol. 2016;212(3):349-364.

21. Dupont S, et al. Role of YAP/TAZ in mechanotransduction. Nature. 2011;474(7350):179-183.

22. Halder G, Dupont S, Piccolo S. Transduction of mechanical and cytoskeletal cues by YAP and TAZ. Nat Rev Mol Cell Biol. 2012;13(9):591-600.

23. Sun Y, Chen CS, Fu J. Forcing stem cells to behave: a biophysical perspective of the cellular microenvironment. Annu Rev Biophys. 2012;41:519-542.

24. Lei QY, et al. TAZ promotes cell proliferation and epithelial-mesenchymal transition and is inhibited by the hippo pathway. Mol Cell Biol. 2008;28(7):2426-2436.

25. Zhou X, Chadarevian JP, Ruiz B, Ying QL. Cytoplasmic and nuclear TAZ exert distinct functions in regulating primed pluripotency. Stem Cell Reports. 2017;9(3):732-741.

26.Ransom RC, et al. Mechanoresponsive stem cells acquire neural crest fate in jaw regeneration. Nature. 2018;563(7732):514-521.

27. Yoon H, Dehart JP, Murphy JM, Lim ST. Understanding the roles of FAK in cancer: inhibitors, genetic models, and new insights. J Histochem Cytochem. 2015;63(2):114-128.

28. Pineault KM, Song JY, Kozloff KM, Lucas D, 
Wellik DM. Hox11 expressing regional skeletal stem cells are progenitors for osteoblasts, chondrocytes and adipocytes throughout life. Nat Commun. 2019;10(1):3168.

29. O'Rourke M, et al. Evaluating tissue-specific recombination in a Pdgfralpha-CreERT2 transgenic mouse line. PloS one. 2016;11(9):e0162858.

30. Qian C, et al. Stage specific requirement of platelet-derived growth factor receptor- $\alpha$ in embryonic development. PLOS ONE. 2017;12(9):e0184473.

31. Couasnay G, Frey C, Elefteriou F. Promoter Cre-specific genotyping assays for authentication of Cre-driver mouse lines. JBMR Plus. 2019;3(4):e10128.

32. Tucker NR, et al. Diminished PRRX1 expression is associated with increased risk of atrial fibrillation and shortening of the cardiac action potential. Circ Cardiovasc Genet. 2017;10(5):e001902.

33. Brighton CT, Albelda SM. Identification of integrin cell-substratum adhesion receptors on cultured rat bone cells. JOrthop Res. 1992;10(6):766-773.

34. Visconti LA, Yen EH, Johnson RB. Effect of strain on bone nodule formation by rat osteogenic cells in vitro. Arch Oral Biol. 2004;49(6):485-492.

35. Binderman I, Zor U, Kaye AM, Shimshoni Z, Harell A, Sömjen D. The transduction of mechanical force into biochemical events in bone cells may involve activation of phospholipase A2. Calcif Tissue Int. 1988;42(4):261-266.

36. Buckley MJ, et al. Osteoblasts increase their rate of division and align in response to cyclic, mechanical tension in vitro. Bone Miner. 1988;4(3):225-236.

37. Hasegawa S, Sato S, Saito S, Suzuki Y, Brunette DM. Mechanical stretching increases the number of cultured bone cells synthesizing DNA and alters their pattern of protein synthesis. Calcif Tissue Int. 1985;37(4):431-436.

38. Neidlinger-Wilke C, Wilke HJ, Claes L. Cyclic stretching of human osteoblasts affects proliferation and metabolism: a new experimental method and its application. JOrthop Res. 1994;12(1):70-78.

39. Khan MA, et al. Nonsurgically induced disuse muscle atrophy and neuromuscular dysfunction upregulates alpha7 acetylcholine receptors. Can J Physiol Pharmacol. 2014;92(1):1-8.

40. Lloyd SA, Lewis GS, Zhang Y, Paul EM, Donahue HJ. Connexin 43 deficiency attenuates loss of trabecular bone and prevents suppression of cortical bone formation during unloading.J Bone Miner Res. 2012;27(11):2359-2372.

41. Crawford CM, Varghese G, Mani MM, Neff JR. Heterotopic ossification: are range of motion exercises contraindicated? JBurn Care Rehabil. 1986;7(4):323-327.

42. Michelsson JE, Granroth G, Andersson LC. Myositis ossificans following forcible manipulation of the leg. A rabbit model for the study of heterotopic bone formation. J Bone Joint Surg Am 1980;62(5):811-815.

43. Michelsson JE, Rauschning W. Pathogenesis of experimental heterotopic bone formation following temporary forcible exercising of immobilized limbs. Clin Orthop Relat Res. 1983;(176):265-272. 44. Daud O, Sett P, Burr RG, Silver JR. The relation- ship of heterotopic ossification to passive movements in paraplegic patients. Disabil Rehabil. 1993;15(3):114-118.

45. Hong JH, et al. TAZ, a transcriptional modulator of mesenchymal stem cell differentiation. Science. 2005;309(5737):1074-1078.

46. Liu F, et al. Mechanosignaling through YAP and TAZ drives fibroblast activation and fibrosis. Am J Physiol Lung Cell Mol Physiol. 2015;308(4):L344-L357.

47. Hwang JH, et al. Extracellular matrix stiffness regulates osteogenic differentiation through MAPK activation. PLOS ONE. 2015;10(8):e0135519.

48. Zhang X, et al. Dynamic mechanical analysis to assess viscoelasticity of liver tissue in a rat model of nonalcoholic fatty liver disease. Med Eng Phys. 2017;44:79-86.

49. Chen CS, Alonso JL, Ostuni E, Whitesides GM, Ingber DE. Cell shape provides global control of focal adhesion assembly. Biochem Biophys Res Commun. 2003;307(2):355-361.

50. Avilion AA, Nicolis SK, Pevny LH, Perez L, Vivian $\mathrm{N}$, Lovell-Badge R. Multipotent cell lineages in early mouse development depend on SOX2 function. Genes Dev. 2003;17(1):126-140.

51. Umbayev B, et al. Elevated levels of the smal GTPase Cdc42 induces senescence in male rat mesenchymal stem cells. Biogerontology. 2018;19(3-4):287-301.

52. Peterson JR, et al. Direct mouse trauma/ burn model of heterotopic ossification. Journal of visualized experiments: JoVE. 2880;2015(102):e52880.

53. Peterson JR, et al. Treatment of heterotopic ossification through remote ATP hydrolysis. Sci Transl Med. 2014;6(255):255ra132.

54. Peterson JR, et al. Early detection of burn induced heterotopic ossification using transcutaneous Raman spectroscopy. Bone. 2013;54(1):28-34.

55 . Hynes RO. The extracellular matrix: not just pretty fibrils. Science. 2009;326(5957):1216-1219.

56. Lu P, Weaver VM, Werb Z. The extracellular matrix: a dynamic niche in cancer progression. JCell Biol. 2012;196(4):395-406.

57. Engler AJ, Sen S, Sweeney HL, Discher DE. Matrix elasticity directs stem cell lineage specification. Cell. 2006;126(4):677-689.

58. Witkowska-Zimny M, Walenko K, Wrobel E, Mrowka P, Mikulska A, Przybylski J. Effect of substrate stiffness on the osteogenic differentiation of bone marrow stem cells and bone-derived cells. Cell Biol Int. 2013;37(6):608-616

59. Guilak F, Cohen DM, Estes BT, Gimble JM, Liedtke W, Chen CS. Control of stem cell fate by physical interactions with the extracellular matrix. Cell Stem Cell. 2009;5(1):17-26.

60. Rowe RG, Weiss SJ. Navigating ECM barriers at the invasive front: the cancer cell-stroma interface. Annu Rev Cell Dev Biol. 2009;25:567-595.

61. Tang Y, Feinberg T, Keller ET, Li XY, Weiss SJ. Snail/Slug binding interactions with YAP/TAZ control skeletal stem cell self-renewal and differentiation. Nat Cell Biol. 2016;18(9):917-929.

62. Tang Y, et al. MT1-MMP-dependent control of skeletal stem cell commitment via a $\beta 1$-integrin/YAP/ TAZ signaling axis. Dev Cell. 2013;25(4):402-416. 63. Tang Y, Weiss SJ. Snail/Slug-YAP/TAZ complex- es cooperatively regulate mesenchymal stem cell function and bone formation. Cell Cycle. 2017;16(5):399-405.

64. Pan JX, et al. YAP promotes osteogenesis and suppresses adipogenic differentiation by regulating $\beta$-catenin signaling. Bone Res. 2018;6:18.

65. Yao F, et al. SKP2- and OTUD1-regulated non-proteolytic ubiquitination of YAP promotes YAP nuclear localization and activity. Nat Commun. 2018;9(1):2269.

66. Fried A, Shamay A, Wientroub S, Benayahu D. Phenotypic expression of marrow cells when grown on various substrata. J Cell Biochem. 1996;61(2):246-254.

67. Discher DE, Mooney DJ, Zandstra PW. Growth factors, matrices, and forces combine and control stem cells. Science. 2009;324(5935):1673-1677.

68. Cui $\mathrm{Y}$, et al. Cyclic stretching of soft substrates induces spreading and growth. Nat Commun. 2015;6:6333.

69. McBeath R, Pirone DM, Nelson CM, Bhadriraju $\mathrm{K}$, Chen CS. Cell shape, cytoskeletal tension, and RhoA regulate stem cell lineage commitment. Dev Cell. 2004;6(4):483-495.

70. Wang YK, et al. Bone morphogenetic protein-2induced signaling and osteogenesis is regulated by cell shape, RhoA/ROCK, and cytoskeletal tension. Stem Cells Dev. 2012;21(7):1176-1186.

71. Seo J, Kim J. Regulation of Hippo signaling by actin remodeling. BMB Rep. 2018;51(3):151-156.

72. David V, et al. Mechanical loading down-regulates peroxisome proliferator-activated receptor gamma in bone marrow stromal cells and favors osteoblastogenesis at the expense of adipogenesis. Endocrinology. 2007;148(5):2553-2562.

73. Forsberg JA, Potter BK, Polfer EM, Safford SD, Elster EA. Do inflammatory markers portend heterotopic ossification and wound failure in combat wounds? Clin Orthop Relat Res. 2014;472(9):2845-2854.

74. Kraft CT, et al. Trauma-induced heterotopic bone formation and the role of the immune system: A review. J Trauma Acute Care Surg. 2016;80(1):156-165.

75. McTighe S, Chernev I. Intramuscular lipoma: a review of the literature. Orthop Rev (Pavia). 2014;6(4):5618.

76. Chen X, Nadiarynkh O, Plotnikov S, Campagnola PJ. Second harmonic generation microscopy for quantitative analysis of collagen fibrillar structure. Nat Protoc. 2012;7(4):654-669.

77. Boudaoud A, et al. FibrilTool, an ImageJ plug-in to quantify fibrillar structures in raw microscopy images. Nat Protoc. 2014;9(2):457-463.

78. Matera DL, Wang WY, Smith MR, Shikanov A, Baker BM. Fiber density modulates cell spreading in 3D interstitial matrix mimetics. ACS Biomat Sci Eng. 2019;5(6):2965-2975.

79. Brown GCJ, Lim KS, Farrugia BL, Hooper GJ, Woodfield TBF. Covalent incorporation of heparin improves chondrogenesis in photocurable gelatin-methacryloyl hydrogels [published online October 25, 2017]. Macromol Biosci. https://doi. org/10.1002/mabi.201700158.

80. Baker BM, et al. Cell-mediated fibre recruitment drives extracellular matrix mechanosensing in engineered fibrillar microenvironments. Nat Mater. 2015;14(12):1262-1268. 